\title{
Design, Analysis, and Experiment for Rescue Robot with Wheel-Legged Structure
}

\author{
Meng Ning, ${ }^{1,2}$ Bilun Xue, ${ }^{1}$ Zefeng Ma, ${ }^{3}$ Changhong Zhu, ${ }^{1}$ Zihao Liu, \\ Cuncai Zhang, ${ }^{1}$ Yao Wang, ${ }^{1}$ and QiuJu Zhang ${ }^{1,2}$ \\ ${ }^{1}$ School of Mechanical Engineering, Jiangnan University, Wuxi 214122, China \\ ${ }^{2}$ Jiangsu Key Laboratory of Advanced Food Manufacturing Equipment and Technology, Jiangnan University, Wuxi 214122, China \\ ${ }^{3}$ School of Automation Science and Electrical Engineering, Beihang University, Beijing 100191, China \\ Correspondence should be addressed to Meng Ning; ningmeng0608@126.com
}

Received 5 June 2017; Revised 26 September 2017; Accepted 12 October 2017; Published 6 November 2017

Academic Editor: Carlos E. Ugalde-Loo

Copyright (C) 2017 Meng Ning et al. This is an open access article distributed under the Creative Commons Attribution License, which permits unrestricted use, distribution, and reproduction in any medium, provided the original work is properly cited.

\begin{abstract}
A wheel-legged rescue robot design with strong environmental adaptability is proposed. The design presented is aimed at helping rescue workers complete their missions, such as environmental and personnel search, quickly and accurately. So it has broad application prospects. In order to achieve the advantages of simple structure, easy control, small occupation space, and wide motion range, a wheel-legged rescue robot is designed in this paper, and the robot can realize three kinds of motion states, which include wheel state, rotation center lifting process, and leg state. Then the motion states are analyzed in detail, which provides a reference for motion control. Considering the wheel state and leg state share the same structure to contact with the ground, the effect of the stiffness of wheel-legged structure to the motion performance is analyzed. Then the experiment is carried out to prove the feasibility of the structure design. This study offers a design and quantitative analysis for wheel-legged rescue robot. Furthermore, a basis for future control research and engineering applications is established.
\end{abstract}

\section{Introduction}

The complex and dangerous environment after the disaster can cause serious danger to the life safety of rescue workers. Rescue robot can perform many tasks instead of rescue workers, such as environmental monitoring and personnel search, and it can improve the rescue efficiency and information accuracy. So the rescue robot has broad application prospect [1-4]. Because the postdisaster environment is very complex, the adaptability of complex environment is the basic function of the rescue robot.

According to the different movement forms, rescue robots can be divided into four categories: tracked rescue robot [5-8], wheeled rescue robot [9], multilegged rescue robot $[10,11]$, and snake-like rescue robot $[12,13]$. However, the movement environment and function of the above four kinds of robots have some limitations. In order to solve the above problems, many researchers have studied the wheel-legged robot connecting the high obstacle capability of multilegged robot and high mechanical efficiency of wheeled robot. According to the different structure form, wheel-legged structure of the robot can be divided into series structure, similar parallel structure, and simplified structure. The leg of mammals and insects has three parts: thigh (femur), calf (tibia), and foot (tarsus); thus, the leg can be considered as a series mechanism [14]. Many wheellegged structures were designed by mimicking biological leg structure and have the advantages of high flexibility. For example, California Institute of Technology designed a sixwheel-legged mobile robot ATHLETE in 2008. Each leg of this robot has six DOF (degrees of freedom), and each wheellegged can change posture flexibly to adapt to different terrain or across obstacles [15, 16]. Amar et al. [17] designed a wheellegged robot, and each leg has three DOF. An electrical actuator with a ball screw is mounted on each sliding joint. Besides, many scientific research institutions [18-21] have carried out in-depth research on the structure design and performance analysis of this type of wheel-legged robots. 


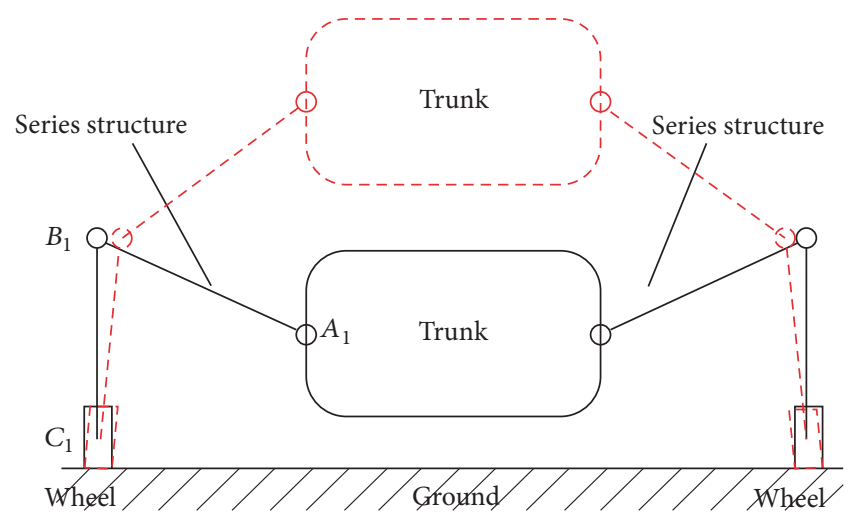

(a)

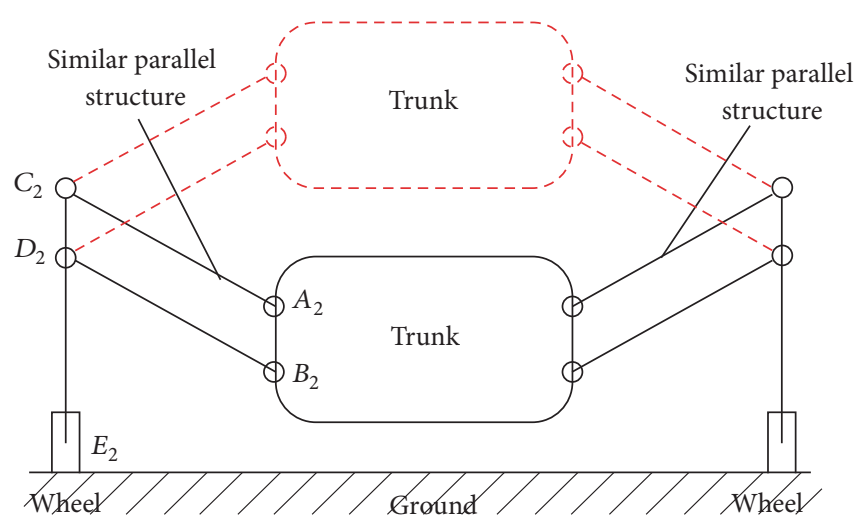

(b)

Figure 1: Common wheel-legged structures. (a) Series wheel-legged structure. (b) Four-bar wheel-legged structure.

Similar parallel structure has the advantages of high load capacity and rigidity, and some research institutions also have studied this type of robot. For example, Wang et al. [22] designed a wheel-legged rescue robot, and the leg is a (2-VPS+U)R series-parallel mechanism. Alamdari et al. [23] explored the use of various candidate articulated legwheel subsystem designs (based on the four-bar mechanism) to enhance locomotion capabilities of land-based vehicles. Besides, Luo et al. [24] and Siegwart et al. [25] also studied this type of wheel-legged robots. For some wheel-legged robots, wheel structure can directly change into leg structure, which is totally different from the biological legs. For example, Kim et al. [26] designed a wheel-leg hybrid robot which utilizes a novel transformable wheel that combines the advantages of both circular and legged wheels. Tadakuma et al. [27] and Chen et al. [28] also have studied this type of robot.

Although many robots with different wheel-legged structures have been designed and the movement performances have been analyzed, the wheel-legged robots still have the disadvantages of complex structure, difficult control, large space required for wheel-legged translation, and limited movement environment. This limits the practical application of the wheel-legged rescue robots. In order to solve the above problems, a new wheel-legged rescue robot is designed and the motion states are analyzed to provide a reference for motion control. Considering the wheel state and leg state share the same structure to contact with the ground, the elastic deformation of the wheel-legged structure is also analyzed. The experimental results prove the feasibility of the structural design and lay the foundation for further research on control methods.

\section{Structure Design}

2.1. Structure Design of Wheel-Legged Rescue Robot. The common series wheel-legged structure and similar parallel structure are shown in Figure 1. In Figure 1(a), link $A_{1} B_{1}$ and link $B_{1} C_{1}$ are connected by hinge, and the wheels are added at the end of the leg. In Figure 1(b), links $A_{1} B_{1}, B_{1} D_{1}, D_{1} C_{1}$, and $C_{1} A_{1}$ consist of four-bar wheel-legged structure. In Figure 1, the solid line and dotted line represent the wheel state and leg state of the robot, respectively. It can be known from Figure 1 that the robot needs a large space when the motion state is converted between the wheel state and leg state, and this limits the application of rescue robot in narrow space.

Considering the arc leg, which is a part of circle, also can make multilegged robot walk, the transformation process from wheel state to leg state is shown in Figure 2. When the left half arc structure and the right half arc structure form a circle, the robot is in the wheel state. And when the left half arc structure rotates around the rotation axis and fits in the right half arc structure, the robot is in the leg state. This kind of wheel-legged structure is simpler than other wheellegged structure, and the transformation process between wheel state and leg state takes up small space. In particular, when the motion process of the robot changes from wheel state to leg state, the rotational center needs to be raised. So the wheel-legged robot has three kinds of motion states: wheel state, rotation center lifting process, and leg state.

The drive schematic of wheel-legged structure is shown in Figure 3. Motor 1 is fixedly connected with gear 1 , and motor 2 is fixedly connected with gear 3 . Gear 1 rotates under the drive of motor 1 . Because gear 2 is engaged with gear 1 , gear 2 is driven to rotate by gear 1 . Gear 2 is connected with the fixing link through connecting plate, and the fixed link fixedly connected with the wheel. So the robot can realize the wheel movement by the driving of motor 1 . Motor 2 is fixedly connected with gear 3 , and gear 3 is engaged with the rack. At this time, the position of the rotation center can be lifted by the driving of motor 2. In particular, the fixed link and rack are fixed to the base plate. So when the robot is in the wheel state, the fixed link rotates, and the rack also rotates. At this time, gear 3 rotates inevitably. Motor 2 should match the motion of motor 1 when the robot is in wheel state. After the rotation center being lifted to specified location, the upper contact point contacts with the lower contact point. The circuit of the motor 3 is connected. Then the left half arc structure rotates by the driving of motor 3 until two half arc structures are fit together. When the rescue robot is in the leg state, the legs swing under the action of motor 1 and motor 2 . The overall structure diagram of wheel-legged rescue robot is shown in Figure 4. 


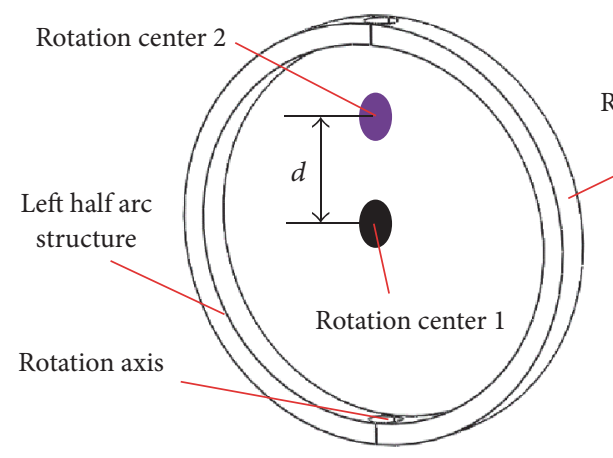

(a)

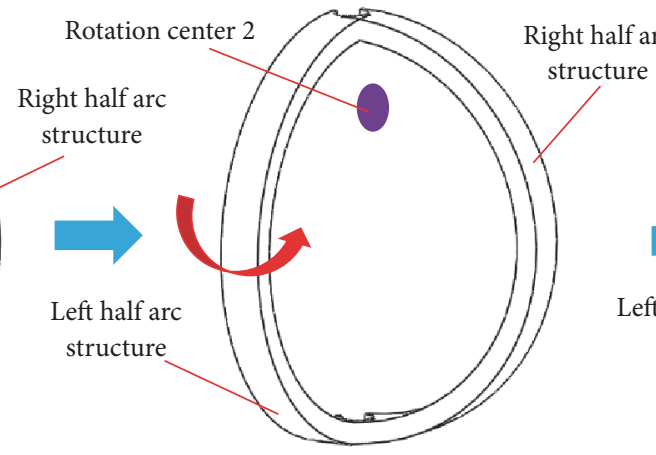

(b)

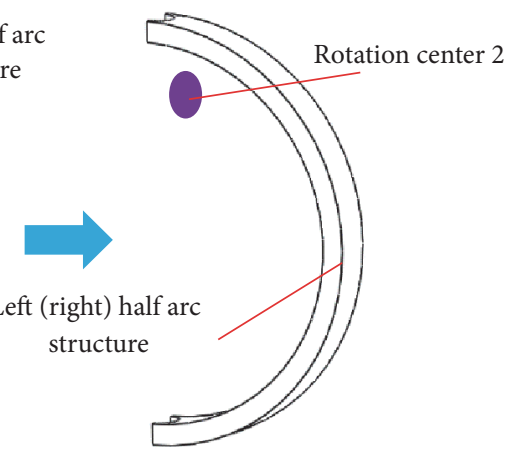

(c)

FIGURE 2: Transformation process from wheel state to leg state. (a) Wheel state. (b) Transformation process. (c) Leg state.

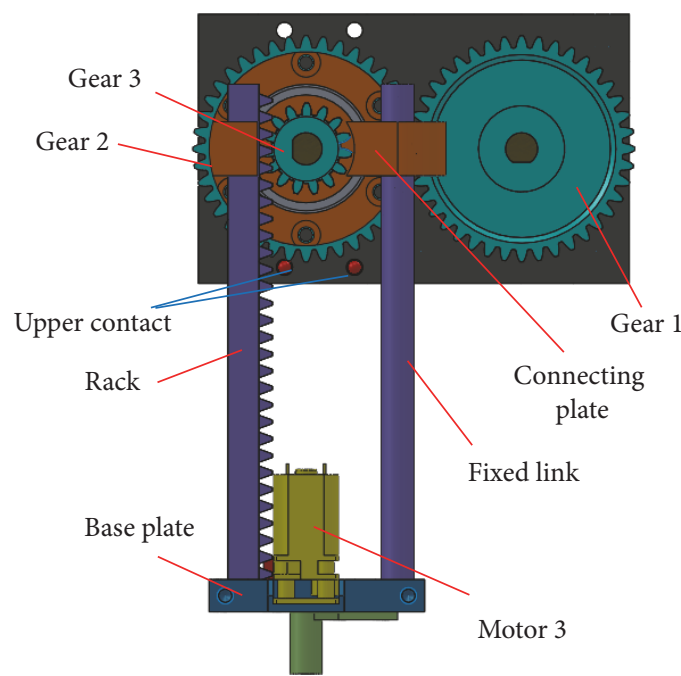

(a)

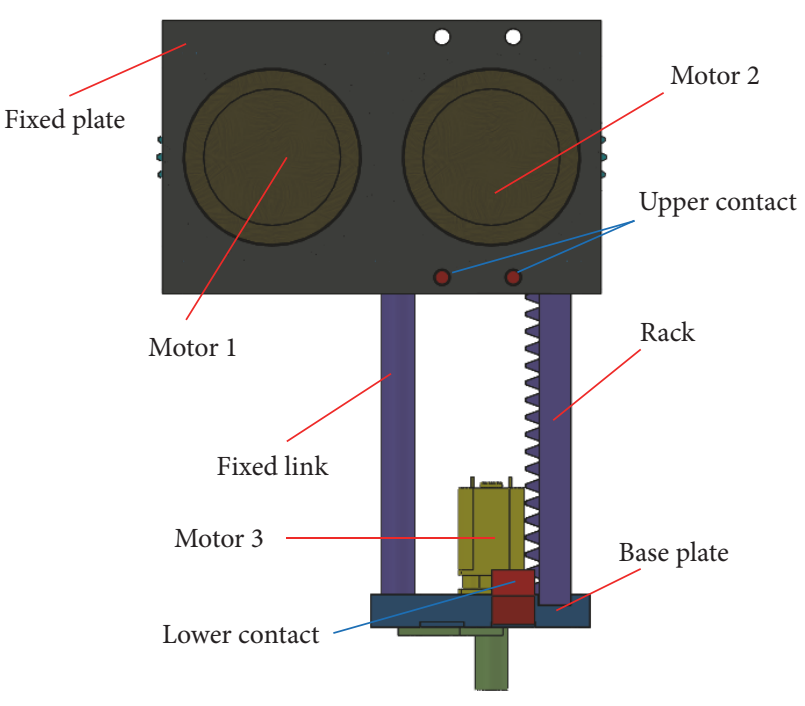

(b)

FIgURE 3: Drive schematic of wheel-legged structure. (a) Main view of main structure. (b) Postview of main structure.

2.2. Application Range of Wheel-Legged Rescue Robot. One of the biggest characteristics of the wheel-legged robot with semicircle legs is that it can not only move on the rugged road (Figure 5(a)), but also move in water (Figure 5(b)). This is what other wheel-legged robots, whether the series wheellegged structure or similar parallel wheel-legged structure, do not have. When the wheel-legged robot moves on land in leg state, the wheel-legged structure can either turn clockwise or counterclockwise. And when the robot moves in the water, the wheel-legged structure can only turn counterclockwise. At this time, the wheel-legged structures are equivalent to the oars. This has greatly broadened the applications scope of robot.

\section{Motion States Analysis}

3.1. Wheel State. The rescue robot needs to overcome all kinds of obstacles, which include road resistance, which is caused by unevenness of the road surface and slope of the ground, and inertia resistance, which is caused by overcoming the inertia of the robot.

Road resistance can be shown as [29]

$$
R_{R}=R_{f}+R_{i}=\mu_{1} \cdot m g+\mu_{2} \cdot \sin \theta \cdot m g,
$$

where $R_{f}$ is the rolling resistance, which is related to the type of road, the velocity, and the structure of the tire. $\mu_{1}$ is the rolling resistance coefficient. $R_{i}$ is the slope resistance. $\theta$ is the angle of slope. $\mu_{2}$ is the slope resistance coefficient, and it is greater than 1 .

Inertia resistance includes the inertia force and inertia moment. The robot is composed of many parts, and the inertia force and inertia moment of each part are not the same. For easy calculation, the inertia resistance can be shown as

$$
R_{I}=\mu_{3} \cdot m a,
$$

where $m$ and $a$ are the total mass and the acceleration of the center of mass, respectively. $\mu_{3}$ is the equivalent coefficient, 


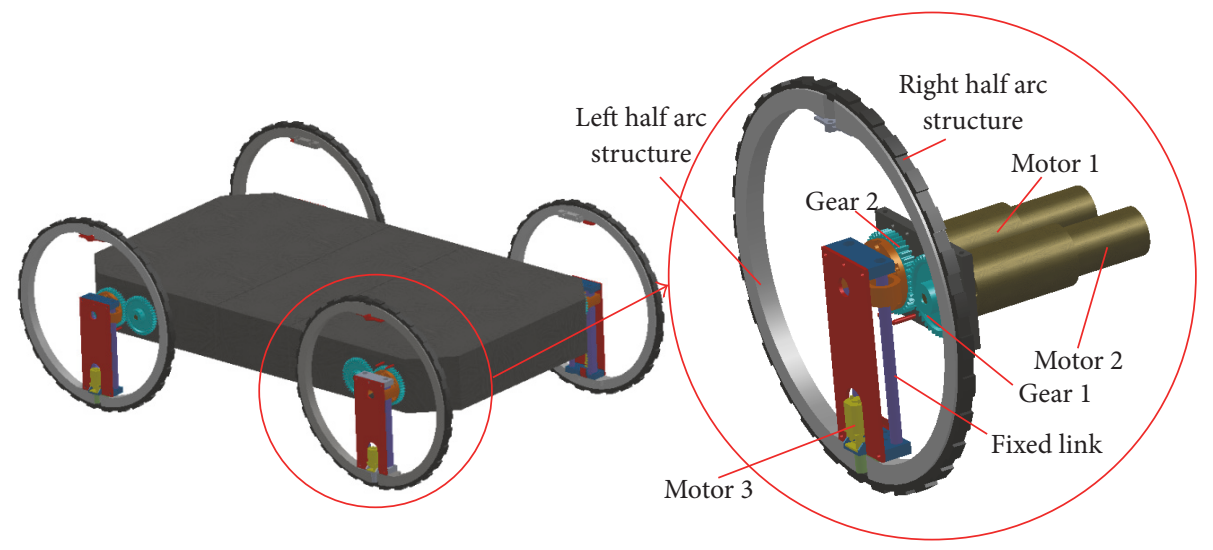

(a)

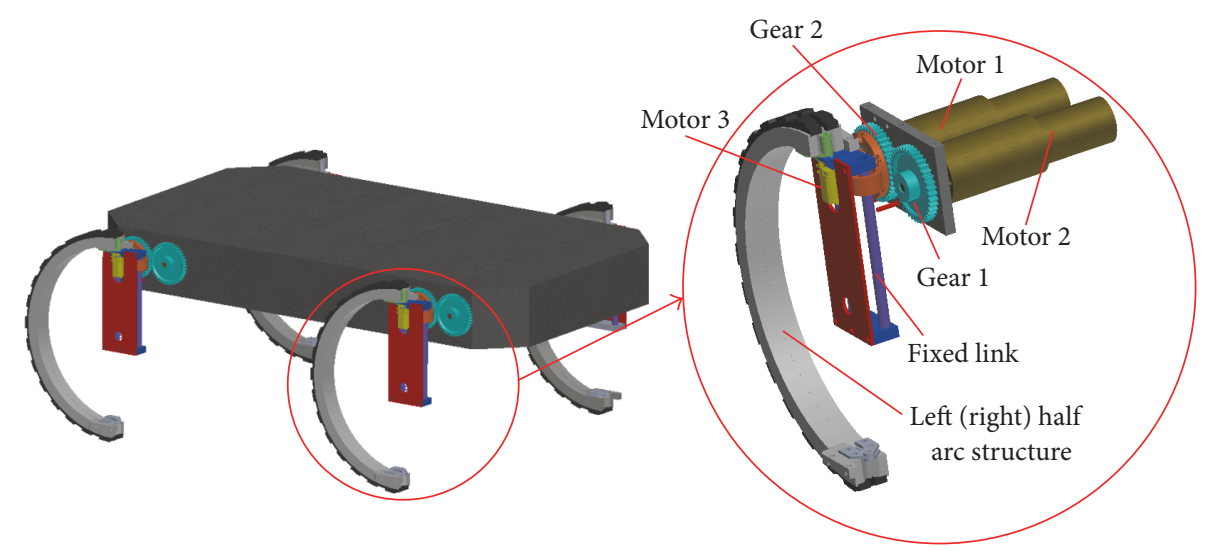

(b)

FIGURE 4: Overall structure diagram of wheel-legged rescue robot. (a) Robot in wheel state. (b) Robot in leg state.

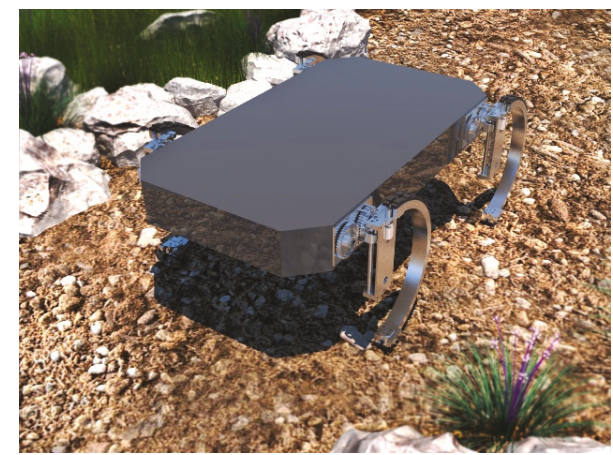

(a)

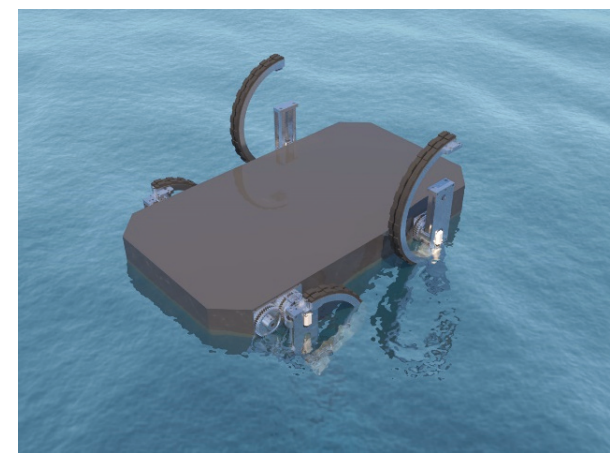

(b)

FIGURE 5: Movement environment of wheel-legged rescue robot. (a) Moving on rough terrain. (b) Moving in the water.

and it is greater than 1 . At this time, the resistance of the wheel-legged rescue robot in wheel state can be shown as

$$
R=R_{R}+R_{I} \text {. }
$$

According to the structural characteristics, the torque of motor 1 can be shown as

$$
M_{1}=\mu_{4} \cdot R \cdot \frac{D}{2} \cdot \frac{i_{1}}{i_{2}},
$$

where $D$ is the diameter of the wheel, $i_{1}$ is the number of teeth of gear 1 , and $i_{2}$ is the number of teeth of gear $2 . \mu_{4}$ is the safety factor, and it is also greater than 1 .

When the rescue robot is in the wheel state, in addition to motor 1, motor 2 also should work at the same time. So the speed of the two motors should be matched. The rotation relationship between motor 1 and motor 2 can be shown in Figure 6. If gear 3 does not rotate and only the rack rotates from position 1 to position 2, the relative position of gear 


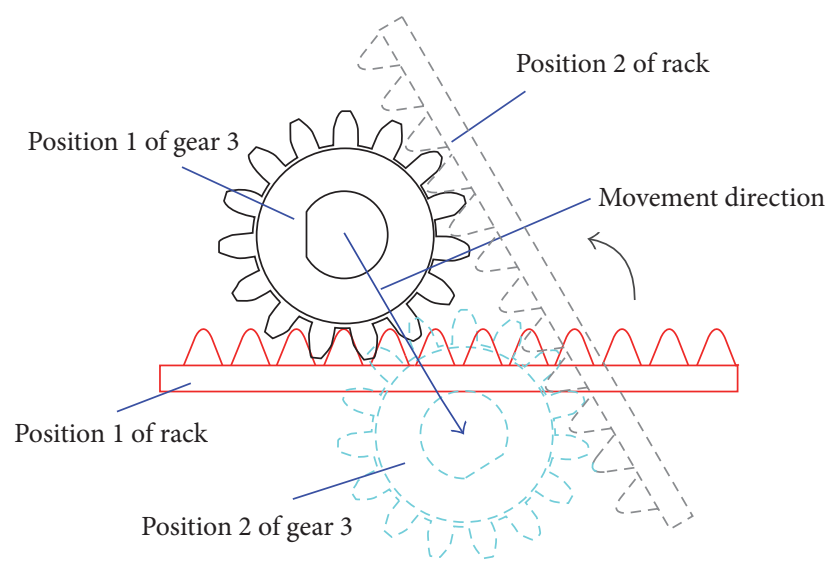

FIGURE 6: Rotation relationship between motor 1 and motor 2 .

and rack changes, which can lead to the rotation center of wheel-legged changes. At this time, the wheel turns into a "cam." According to geometric relationship, the relationship between the speeds of the two motors can be expressed as

$$
n_{3}=n_{1} \cdot \frac{i_{1}}{i_{2}}
$$

where $n_{1}$ is the speed of motor 1 and $n_{3}$ is the speed of motor 3 . The rotation directions of the two motors are the same.

3.2. Rotation Center Changing Process. The rotation center can be changed under the action of motor 2 , and it is also the change process of movement state between wheel state and leg state. When the trunk is lifted, the torque can be shown as

$$
M_{t 2}=\mu_{5} \cdot \frac{1}{4} m g \cdot r_{g 3},
$$

where $m$ is the mass of robot; $r_{g 3}$ is the radius of gear $3 . \mu_{5}$ is the safety factor, and it is greater than 1 . When the trunk moves downwards, motor 2 plays a limiting role. Besides, when the left half arc structure rotates, motor 3 is mainly to overcome the inertia resistance, and the torque of the motor 3 can be calculated according to (3).

3.3. Leg State. There are two walking ways when the robot is in wheel state: arcuate part of the wheel-legged structure touches the ground first and endpoint of the wheel-legged structure touches the ground first. For the former, the motion sequence can be shown in Figure 7(a). When the robot walks on the horizontal ground, the midpoint of the semicircle arc lands first, and then the structure rotates clockwise until the end point contacts with the ground. This movement mode is suitable for the robot to move on a relatively flat ground, and it has the advantage of high efficiency. For the latter, the motion sequence can be shown in Figure 7(b). The endpoint of the semicircle arc lands first, and then the structure rotates around the landing point until the diameter is perpendicular to the ground. This movement mode is suitable for the stepped road because the endpoint of the semicircle arc is not easy to slide.
In order to analyze the driving torque of the wheel-legged structure when the robot is in the walking process, force analysis should be conducted. For the first walking way, the moment balance equation is

$$
\mathbf{M}+\mathbf{F}_{S 1} \times \mathbf{d}_{S 1}+m_{l} \mathbf{g} \times \mathbf{d}_{S 2}+\mathbf{F}_{c 1} \times \mathbf{d}_{c 1}+\mathbf{M}_{c 1}=0,
$$

where $\mathbf{M}$ is the driving torque, $\mathbf{F}_{S 1}$ is the force of the trunk to the leg, and $m_{l}$ is the mass of the wheel-legged structure. $\mathbf{F}_{c 1}$ and $\mathbf{M}_{c 1}$ are inertia force and inertia moment, respectively. $\mathbf{d}$ is the vector from the point of force to the contact point between the wheel-legged structure and the ground. To simplify the calculation process, consider the speed of the leg in the swing phase is large and the speed of the leg in support phase is small, regardless of the inertia force and inertia moment. And safety factor can be introduced. According to the geometric relationship, (7) can be simplified as

$$
M=\mu_{6}\left[F_{S 1} \cdot r \cos \alpha+m_{l} g \cdot l_{S 1} \cdot \cos \left(\frac{\pi}{2}-\beta\right)\right],
$$

where $l_{S 1}=r \sqrt{2-2 \cos \alpha} ; \beta=\sin \alpha / \sqrt{2-2 \cos \alpha} . r$ is the radius of the wheel-legged structure, and $\alpha$ is rotation angle of leg. $\mu_{6}$ is the safety factor.

For the second walking way, the moment balance equation is the same as (7). In particular, gravity always does negative work for the first walking way, and gravity does positive work and then does negative work for the second walking way. The driving torque of the robot with the second walking way can be shown as

$$
M=F_{S 1} \cdot 2 r \cos \alpha+m_{s} g \cdot \sqrt{2} r \cdot \sin \left(\frac{\pi}{4}-\alpha\right) .
$$

The initial angle can be expressed as

$$
\alpha_{0}=\arcsin \frac{h}{r}
$$

where $h$ is the height of step. Suppose the mass of trunk of robot is $40 \mathrm{Kg}, m_{l}=0.5 \mathrm{Kg}, h=0.1 \mathrm{~m}$, and $r=0.2 \mathrm{~m}$. The variation law of driving force is shown in Figure 8. Horizontal axis represents the rotation angle, and vertical coordinate represents torque. It can be known from Figure 8 that average torque of the robot in the second walking way is greater than average torque in the first walking way.

After knowing the driving torque, the motion speed of the robot also can be obtained. For the first walking way, the relationship between motor speed and motion speed of robot is

$$
\begin{aligned}
& v_{x}=r \dot{\theta} \sin \theta+r \\
& v_{y}=r \dot{\theta} \cos \theta
\end{aligned}
$$

For the second walking way, the relationship between motor speed and motion speed of robot can be shown as

$$
\begin{aligned}
& v_{x}=2 r \dot{\theta} \sin \theta \\
& v_{y}=2 r \dot{\theta} \cos \theta .
\end{aligned}
$$




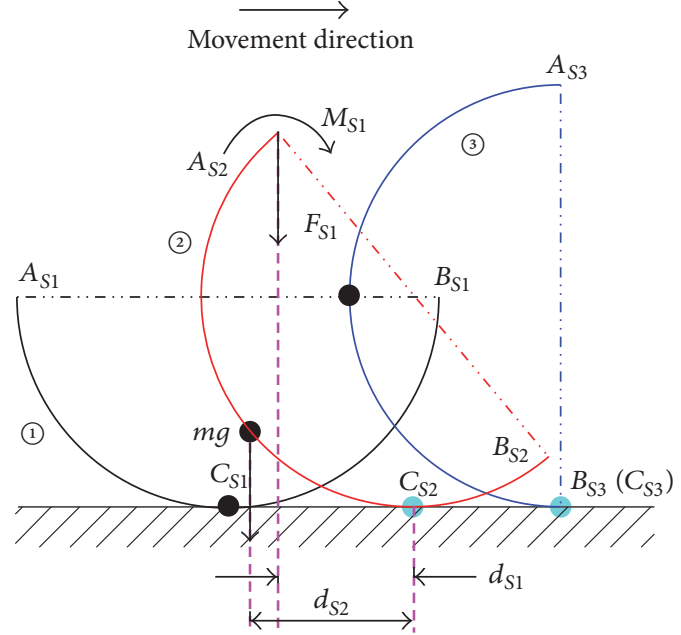

(a)

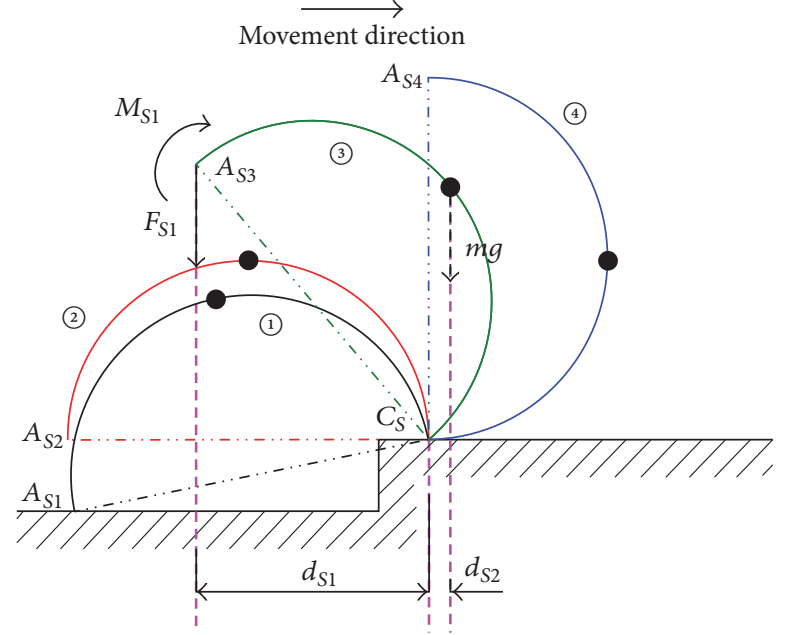

(b)

FIGURE 7: Walking ways of the rescue robot. (a) Arcuate part of the wheel-legged structure lands first. (b) Endpoint of the wheel-legged structure lands first.

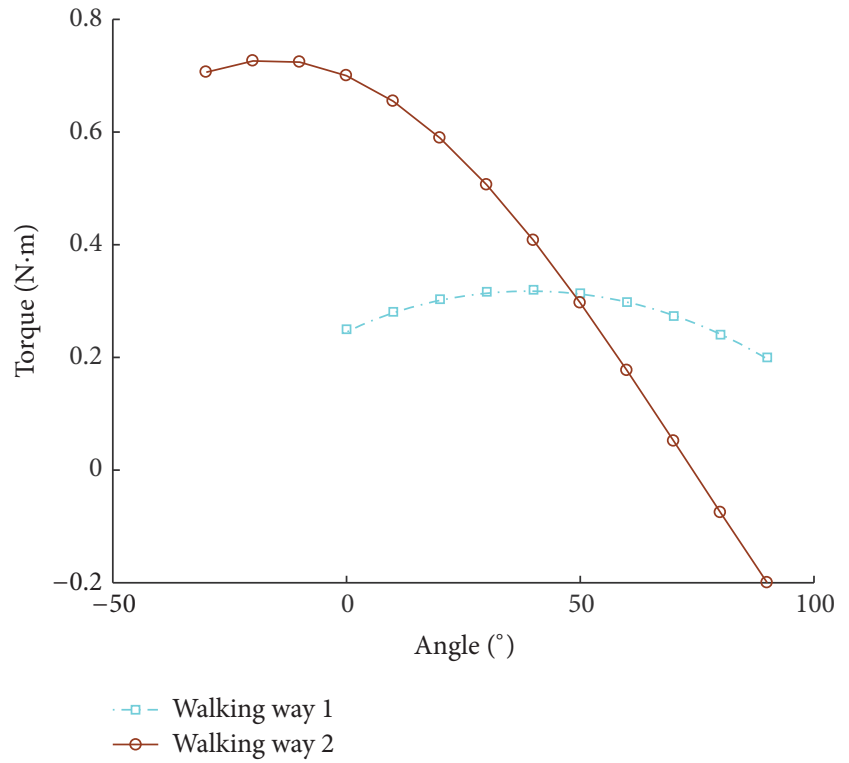

FIgURE 8: Variation law of driving torque in leg state.

When the speed of motor is $1 \mathrm{rad} / \mathrm{s}$, the relationship between the rotation angle of the wheel-legged structure and the speed of the robot can be shown in Figure 9. In the leg state, the robot has both the speed along the horizontal direction and the speed along the vertical direction. When the robot moves in the second walking way, the speed along the vertical direction is greater, and this also shows that the robot is more suitable for ladder-type pavement with the second walking way.

Based on the analysis results of the wheel state, rotation center changing process, and leg state, motion law of wheellegged structure and motor torques can be obtained. In different motion states, the required motor torque is also

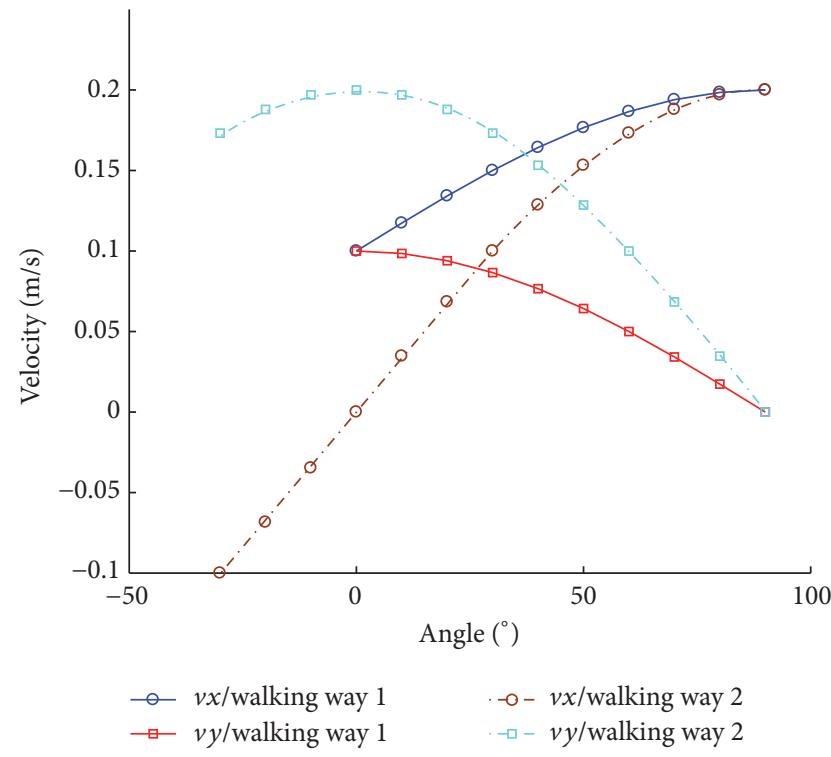

FIGURE 9: Variation law of speed in leg state.

different. After a comprehensive consideration, the model of motor 1 and motor 2 is maxon RE35, and the rated torque is $2.5 \mathrm{Nm}$. The model of motor 3 is GA12YN20, and the rated torque is $0.7 \mathrm{Nm}$.

In particular, when the robot is in leg state, it also can move in the water. At this time, each leg is affected by the force of water on it. The force balance equation can be shown as

$$
\begin{array}{r}
\mathbf{F}_{d}+\mathbf{F}_{c d}+\mathbf{F}_{t}=0 \\
\mathbf{l}_{d \perp} \times \mathbf{F}_{d}+\mathbf{M}_{c d}+\mathbf{M}_{d}=0,
\end{array}
$$

where $\mathbf{M}_{d}$ is motor torque, $\mathbf{M}_{c d}$ and $\mathbf{F}_{c d}$ are inertia force and inertia moment, respectively, $\mathbf{F}_{d}$ is the force of water on 
wheel-legged structure, $\mathbf{F}_{t}$ is the force of trunk on wheellegged structure, and $\mathbf{l}_{d \perp}$ is acting arm. For the wheel-legged rescue robot, it suffers friction, pressure difference resistance, wave resistance, and air resistance. Because the velocity is not great, the friction of water and air resistance is very small. The pressure difference resistance is proportional to the square of the velocity of robot. The wave resistance is introduced due to the wave of water. In order to simplify the calculation, it can be considered proportional to the speed of the robot. The force balance equation of robot can be written as

$$
\left(m_{d 1}+m_{d 2}\right) \frac{d v_{d}}{d t}=-a v_{d}-k v_{d}^{2}+F_{d}
$$

where $m_{d 1}$ is the mass of robot, $m_{d 2}$ is the mass of load, and $v_{d}$ is the velocity of robot. $a$ and $k$ are coefficients of wave resistance and pressure difference resistance, respectively. $F_{d}$ is the force of the water to the robot. The calculation method of $F_{d}$ is different when the underwater environment is different. For example, the wave force in the regular wave can be written as [30]

$$
F_{d}=\rho V\left[1+K_{33} \frac{\omega_{e}}{\omega}\right] \omega^{2} k_{1} e^{\left(-\omega^{2} / g\right) H} \zeta_{a} e^{i \omega_{e} t},
$$

where $\rho$ is the density of water, $V$ is the drainage volume, and $K_{33}$ is the vertical and longitudinal additional mass coefficients. $\omega_{e}$ and $\omega$ are encounter frequency and wave circle frequency, respectively. $k_{1}$ is reduction coefficient, which represents the influence of the ratio of the length of the underwater robot and the effective wavelength on the wave force. $\zeta_{a}$ is amplitude of regular wave.

\section{Structural Stiffness Analysis}

Because the disaster environment is very complex, the rescue robot should have good buffering performance. Otherwise, whether the robot is in the wheel state or in the leg state, the wheel-legged structures of the robot may have rigid collision with the ground frequently and further lead to a series of problems. Considering the wheel state and leg state share the same structure to contact with the ground, the effect of structural stiffness of wheel-legged structure in wheel state and leg state on motion performance is analyzed.

4.1. Structural Stiffness in Wheel State. The wheel should be elastic wheel, which means that hub should be able to achieve deformation under external force. In addition to the elastic deformation of the wheel, the tire can also achieve elastic deformation, so the motion of the robot on the rough road surface can be simplified to a two-DOF vibration system in the vertical direction.

According to Lagrange equation, motion equations of equivalent vibration system can be expressed as [31,32]

$$
\begin{aligned}
& m_{2} \ddot{z}_{2}=k\left(z_{1}-z_{2}\right)+c\left(\dot{z}_{1}-\dot{z}_{2}\right) \\
& m_{1} \ddot{z}_{1}=c\left(\dot{z}_{2}-\dot{z}_{1}\right)+k\left(z_{2}-z_{1}\right)-k_{t}\left(z_{1}-q\right),
\end{aligned}
$$

where $z_{1}$ and $z_{2}$ are vertical displacement of wheel and body, $m_{2}$ is the mass of robot, $k$ is the equivalent stiffness coefficient of wheel, $c$ is the damping of wheel, $m_{1}$ is the mass of wheel, and $k_{t}$ is the equivalent stiffness coefficient of tyre. $q$ is the input of road roughness, and it is harmonic exciting force. The output can be written as $z_{1}=z_{10} e^{i \omega t+\varphi}$ and $z_{2}=z_{20} e^{i \omega t+\varphi}$. Inserting them into (16), it can be written as

$$
\begin{aligned}
z_{2}\left(-\omega^{2} m_{2}+i \omega c+k\right) & =z_{1}(i \omega c+k) \\
z_{1}\left(-\omega^{2} m_{1}+i \omega c+k+k_{t}\right) & =z_{2}(i \omega c+k)+q k_{t} .
\end{aligned}
$$

At this time, the frequency response function can be shown as

$$
\begin{aligned}
& \frac{z_{2}}{z_{1}}=\frac{i \omega c+k}{-\omega^{2} m_{2}+i \omega c+k} \\
& \frac{z_{1}}{q}=\frac{A_{2} k_{t}}{A_{3} A_{2}-A_{1}^{2}} \\
& \frac{z_{2}}{q}=\frac{z_{2}}{z_{1}} \cdot \frac{z_{1}}{q}=\frac{A_{1} k_{t}}{A_{3} A_{2}-A_{1}^{2}},
\end{aligned}
$$

where $A_{1}=i \omega c+k ; A_{2}=-\omega^{2} m_{2}+i \omega c+k ; A_{3}=-\omega^{2} m_{1}+$ $i \omega c+k+k_{t}$. The amplitude frequency characteristics of the robot relative to road surface are obtained:

$$
\left|\frac{z_{2}}{q}\right|=\gamma \sqrt{\frac{\left(1-\lambda^{2}\right)^{2}+(2 \xi \lambda)^{2}}{\Delta}},
$$

where $\Delta=\left[\left(1-\lambda^{2}\right)\left(1+\gamma-\lambda^{2} / \mu\right)^{2}-1\right]+(2 \lambda \xi)^{2}\left[\gamma-(1 / \mu+1) \lambda^{2}\right]^{2}$. $\lambda$ is the frequency ratio, $\mu$ is mass ratio of body and wheel, and $\xi$ is damping ratio. According to the calculation results of the three-dimensional software (Solidworks), $m_{1}=0.5 \mathrm{Kg}$, $m_{2}=40 \mathrm{Kg}$. According to the vibration model of the car, $k_{t}$ $=200000 \mathrm{~N} / \mathrm{m}$ [33]. The amplitude frequency characteristics of robot body with different $k$ can be shown in Figure 10(a). As can be seen from Figure 10(a), the reduction of the spring stiffness coefficient can effectively reduce the resonance peak. But the increase of the spring stiffness coefficient can shock absorption. Amplitude frequency characteristics of body acceleration to road surface velocity can be written as

$$
\left|\frac{\ddot{z}_{2}}{\dot{q}}\right|=\left|\frac{z_{2} \omega^{2}}{q \omega}\right|=\omega\left|\frac{z_{2}}{q}\right| .
$$

The amplitude frequency characteristics of relative dynamic load on road surface velocity can be shown as

$$
\frac{F_{d}}{G \dot{q}}=\frac{m_{2} \ddot{z}_{2}}{m_{2} g \dot{q}}=\frac{1}{g} \frac{\ddot{z}_{2}}{\dot{q}} .
$$

The amplitude frequency characteristics of body acceleration to road surface velocity and relative dynamic load on road surface velocity can be shown in Figures 10(b) and $10(c)$, respectively. The trends are almost the same. In lowfrequency section and resonance section, the bigger $k$ is, the better the ride comfort is. And in high frequency section, the smaller $k$ is, the better the ride comfort is.

It can be known from Figure 10 that the decrease of stiffness coefficient can effectively reduce the resonance peak. 


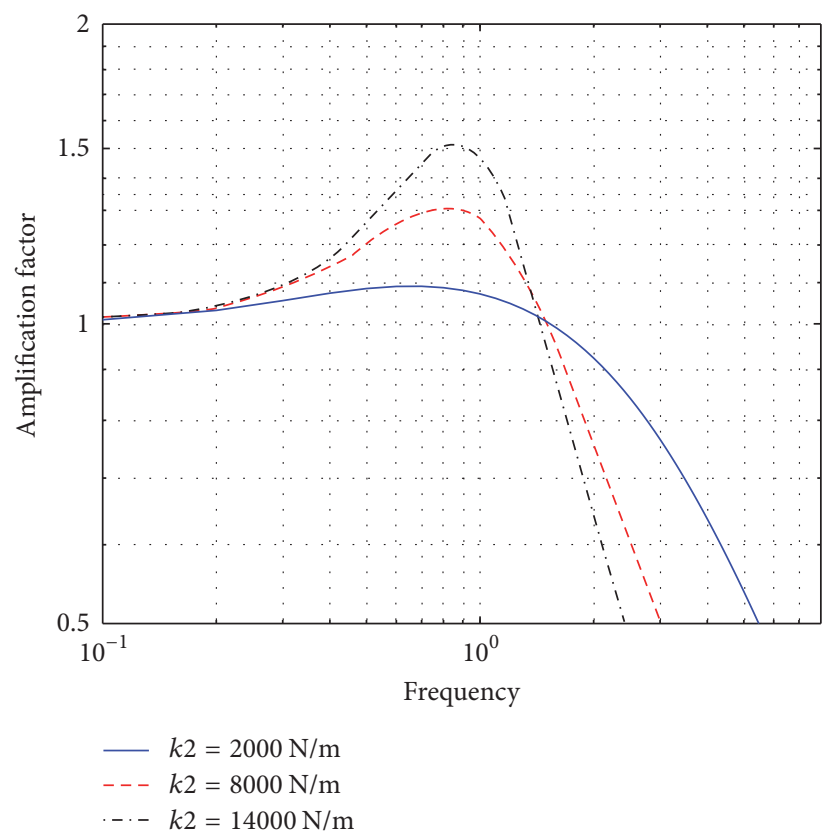

(a)

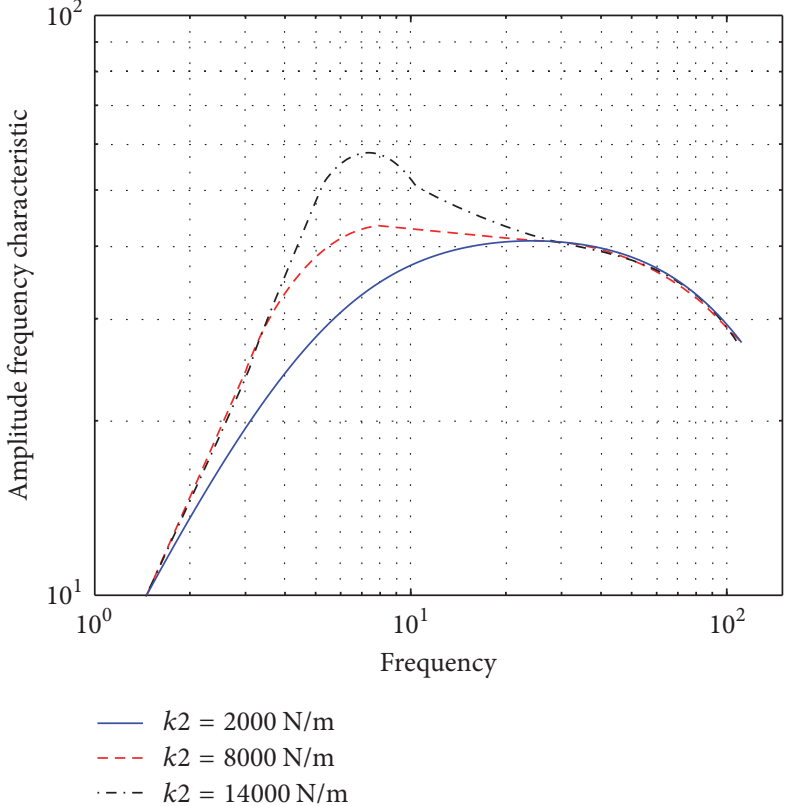

(b)

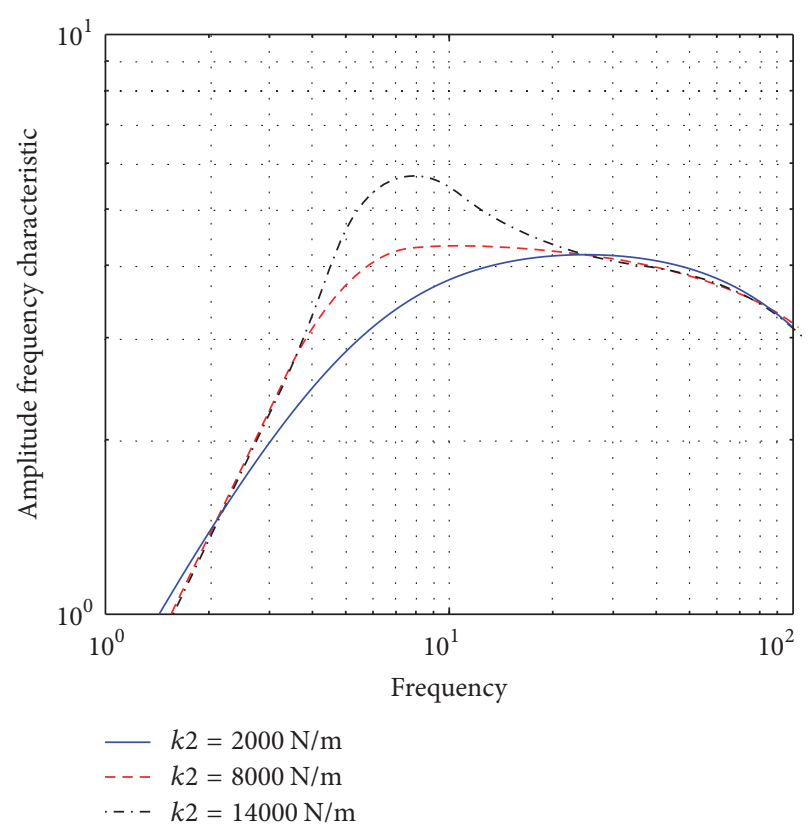

(c)

Figure 10: Amplitude frequency characteristics of equivalent vibration system. (a) Amplitude frequency characteristics of displacement input and displacement output. (b) Amplitude frequency characteristics of body acceleration to road surface velocity. (c) Amplitude frequency characteristics of relative dynamic load on road surface velocity.

But it may reduce shock absorption effect. Besides, the smaller stiffness may produce greater friction because the wheel deformation can make the contact of the wheel with the ground change from point contact to line contact.

4.2. Structural Stiffness in Leg State. When the rescue robot is in leg state, the buffering performance is also important. The arc leg is a compliant mechanism, and it is hard to analyze the buffering performance because the arc leg introduces geometric nonlinearities. The pseudo-rigid-body model used in this study can simplify large-deflection analysis [34-36]. For the first walking way (the midpoint of the arc leg lands first), the leg structure which plays a role in buffering can be considered to be $1 / 4$ circles. At this time, the $2 \mathrm{R}$ model is used, and the pseudo-rigid-body model is shown in Figure 11(a). The equivalent rotation pair at $A_{L 2}$ is $R_{L 11}$, and equivalent 


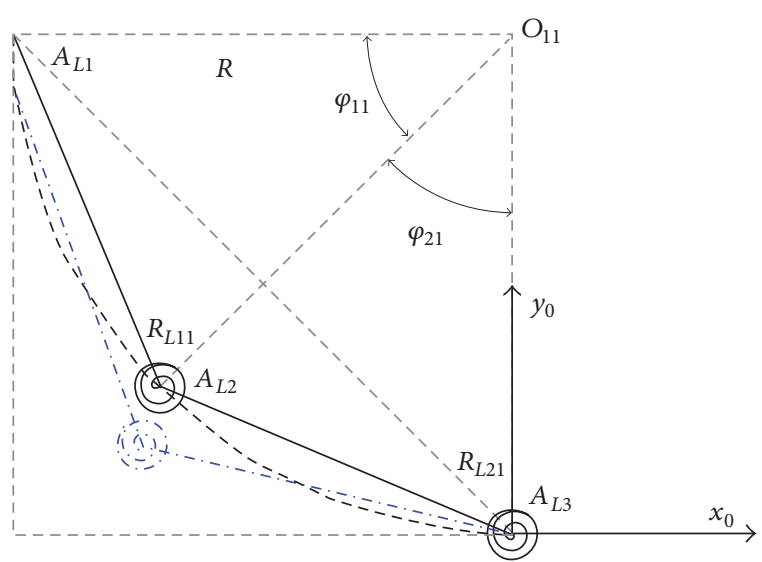

(a)

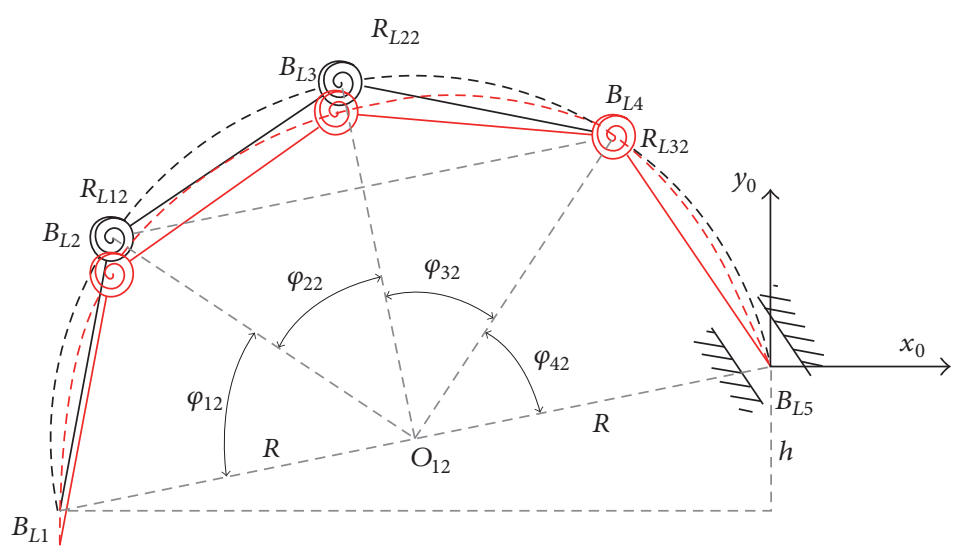

(b)

FIGURE 11: Pseudo-rigid-body model of arc leg. (a) First walking way. (b) Second walking way.

rotation pair at $A_{L 3}$ is $R_{L 21} \cdot A_{L 1}$ is the connection point between the trunk and the leg, and $A_{L 3}$ is the landing point. So the arc leg can be equivalent to 2-DOF serial mechanism, and torsion spring is used in each rotation pair. The coordinate origin of the fixed coordinate system $o_{0}-x_{0} y_{0}$ coincides with the landing point. The direction of the $x_{0}$-axis is horizontal to the right, and the direction of the $y_{0}$-axis is perpendicular to the ground and is upward.

Suppose the trunk moves only in the vertical direction. The coordinate of $A_{L 1}$ is $\left(-R, R-h_{1}\right)$. In triangle $A_{L 1} A_{L 2} A_{L 3}$, the length of $A_{L 1} A_{L 3}$ can be shown as

$$
s=\sqrt{\left(R-h_{1}\right)^{2}+R^{2}} .
$$

The rotation angles of link $A_{L 1} A_{L 2}$ relative to link $A_{L 2} A_{L 3}$ can be shown as

$$
\gamma_{1}=\pi-\arccos \frac{l_{11}^{2}+l_{21}^{2}-s^{2}}{2 \cdot l_{11} \cdot l_{21}}-\gamma_{10} .
$$

The rotation angles of link $A_{L 2} A_{L 3}$ relative to horizontal direction can be written as

$$
\gamma_{2}=\arctan \frac{R-h_{1}}{R}-\arccos \frac{l_{21}^{2}+s^{2}-l_{11}^{2}}{2 \cdot l_{21} \cdot s}-\gamma_{20},
$$

where $h_{1}$ is the trunk movement distance, $R$ is the radius of wheel-legged structure, and $l_{11}$ and $l_{21}$ are the length of link $A_{L 1} A_{L 2}$ and link $A_{L 2} A_{L 3} \cdot \gamma_{10}$ and $\gamma_{20}$ are initial angles. In order to obtain the mechanical properties of buffering process, the force analysis also can be conducted. The force and torque balance equation of each link can be shown as

$$
\begin{aligned}
& \mathbf{F}_{i}-\mathbf{F}_{i+1}+\mathbf{F}_{c i}+\mathbf{m}_{i} \mathbf{g}=0 \\
& \mathbf{M}_{i}-\mathbf{M}_{i+1}-\mathbf{r}_{i+1} \times \mathbf{F}_{i+1}+\mathbf{r}_{i} \times \mathbf{F}_{i}+\mathbf{r}_{c i} \times\left(\mathbf{F}_{c i}+\mathbf{m}_{i} \mathbf{g}\right) \\
& \quad=0,
\end{aligned}
$$

where $\mathbf{r}$ is the vector of the coordinate system origin to the point of force. $\mathbf{F}_{i}$ and $\mathbf{M}_{i}$ are the force and moment of link $l_{i-1}$ to $l_{i}$, and $\mathbf{F}_{i+1}$ and $\mathbf{M}_{i+1}$ are the force and moment of link $l_{i+1}$ to $l_{i} . \mathbf{F}_{c i}$ and $\mathbf{M}_{c i}$ are the inertia force and inertia moment, respectively. When $i=2$ or $3, \mathbf{M}_{i}$ represents the torque generated by the torsion springs. When $i=3, \mathbf{F}_{3}$ represents the force of the ground to the leg. To simplify the calculation process, regardless of the mass of the legs, the mass of the robot remains concentrated in the trunk. Spring damping is also not considered. At this time, the support force of the ground to the wheel-legged structure can be written as

$$
\left[\begin{array}{c}
F_{x} \\
F_{y}
\end{array}\right]=\left[\begin{array}{cc}
-l_{1} \cdot \sin \gamma_{1} & l_{1} \cdot \cos \gamma_{1} \\
l_{2} \cdot \sin \gamma_{2} & l_{2} \cdot \cos \gamma_{2}
\end{array}\right]^{-1}\left[\begin{array}{c}
M_{1}-M_{2} \\
M_{2}+M_{3}
\end{array}\right],
$$

where $M_{2}=k_{11} \cdot \gamma_{11}, M_{3}=k_{21} \cdot \gamma_{21} \cdot k_{i 1}$ is the torsional stiffness coefficient.

For the second walking way, the pseudo-rigid-body model is shown in Figure 11(b). The equivalent rotation pairs at $B_{L 2}, B_{L 3}$, and $B_{L 4}$ are $R_{L 12}, R_{L 22}$, and $R_{L 32}$, respectively. For the $1 / 4$ circles, it is still the 2 -DOF serial mechanism. In particular, considering the complexity of the environment, the end point of the leg is likely to fall into the soft soil or crevice. So $B_{L 4}$ can be seen as fixed point. The attitude of link $B_{L 1} B_{L 2}$ is unchanged in buffering process.

In triangle $B_{L 2} B_{L 3} B_{L 4}$, the length of $B_{L 2} B_{L 4}$ can be shown as

$$
s=\sqrt{T_{1}^{2}+T_{2}^{2}}
$$

where $T_{1}=l_{4} \cdot \sin \gamma_{40}-l_{1} \cdot \sin \gamma_{10}-h-h_{1} ; T_{2}=2 R \cdot \cos \varphi-$ $l_{4} \cdot \cos \gamma_{40}-l_{1} \cdot \cos \gamma_{10} \cdot l_{i}$ is the length of $B_{L i} B_{L(i+1)} \cdot \theta_{10}$ is the angle between link $B_{L 1} B_{L 2}$ and $x_{0}$-axis, and $\theta_{40}$ is the angle between link $B_{L 4} B_{L 5}$ and $x_{0}$-axis. $\varphi$ is the initial inclination angle of wheel-legged structure.

At this time, the rotation angles of link $l_{(i-1) 1}$ relative to link $l_{i 1}$ can be shown as

$$
\gamma_{2}=\pi-\arccos \frac{l_{22}^{2}+l_{32}^{2}-s^{2}}{2 \cdot l_{22} \cdot l_{32}}-\gamma_{20}
$$




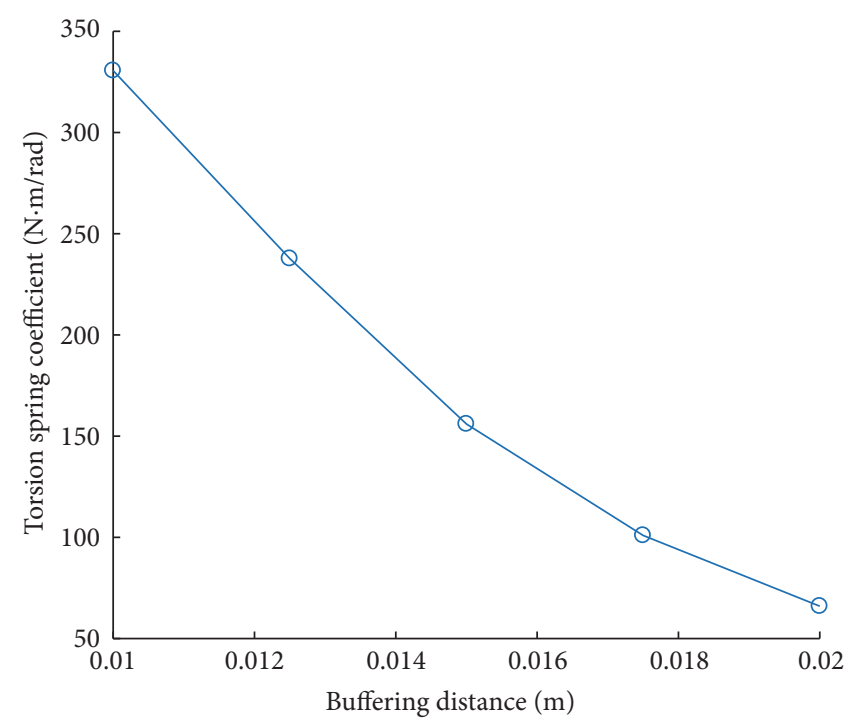

FIGURE 12: Relationship between $h$ and $k_{1}$.

$$
\begin{aligned}
\gamma_{3} & =\pi \\
& -\left(\arccos \frac{l_{32}{ }^{2}+s^{2}-l_{22}^{2}}{2 \cdot l_{32} \cdot s}+\arctan \frac{T_{2}}{T_{1}}+\frac{\pi}{2}-\theta_{40}\right) \\
& -\gamma_{30}
\end{aligned}
$$

$$
s s=\sqrt{\left(l_{4} \cdot \sin \theta_{40}+l_{3} \cdot \sin \theta_{3}+h+h_{1}\right)^{2}+\left(2 R \cdot \cos \varphi-l_{4} \cdot \cos \theta_{40}-l_{3} \cdot \cos \theta_{3}\right)^{2}} .
$$

where

$\theta_{3}$ is the angle between link $B_{L 3} B_{L 4}$ and $x_{0}$ axis.

According to (25)-(26), the support force of the ground to the leg can be obtained.

In order to analyze the mechanical properties, the stiffness coefficient of equivalent torsion springs should be further determined. When the external forces the wheellegged structure suffers are the same and the deformation is also the same, the relationship between equivalent torsion spring stiffness coefficient and equivalent stiffness coefficient of leg can be determined according to experiment.

If the landing ground is rigid, $B_{L 4}$ can be seen as fixed point. It can be simplified as a revolute pair. So pseudorigid-body model has four DOF. At this time, the kinematics can be analyzed according to the same method. Because the equivalent model has four DOF and only 3 constraints can be contained, including the position and posture of end link, the angle of each link cannot be determined directly, and kinematic relationship can be written as

$$
\left(\begin{array}{lll}
\gamma_{1} & \gamma_{2} & \gamma_{3}
\end{array}\right)=f_{i}\left(\gamma_{4}\right)
$$

Equation (33) shows that $\gamma_{1}, \gamma_{2}$, and $\gamma_{3}$ can be expressed with $\gamma_{4}$. In order to further solve $\gamma_{4}$, the force balance equation can be added according to (25)-(26). Considering kinematics

$$
\gamma_{1}=\arccos \frac{l_{12}{ }^{2}+l_{22}{ }^{2}-s s^{2}}{2 \cdot l_{12} \cdot l_{22}}-\gamma_{10}
$$




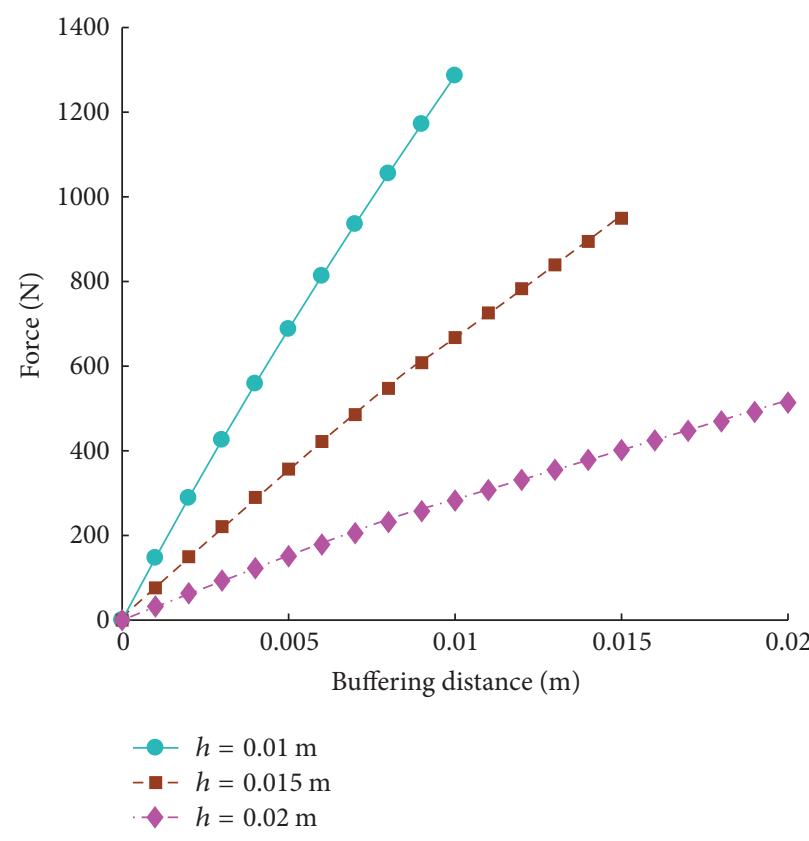

(a)

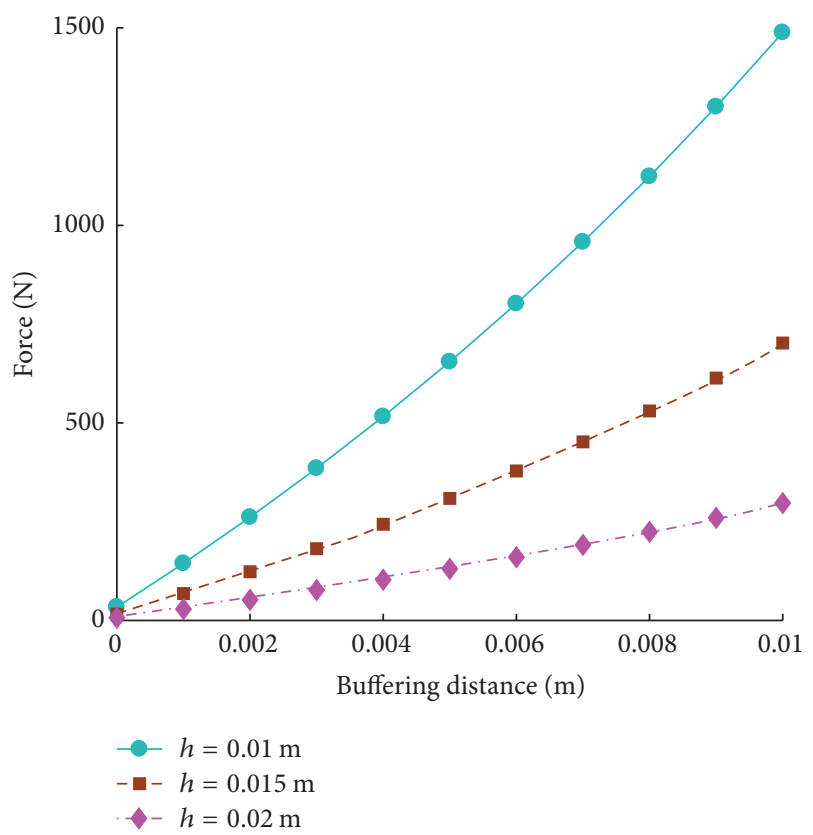

(c)

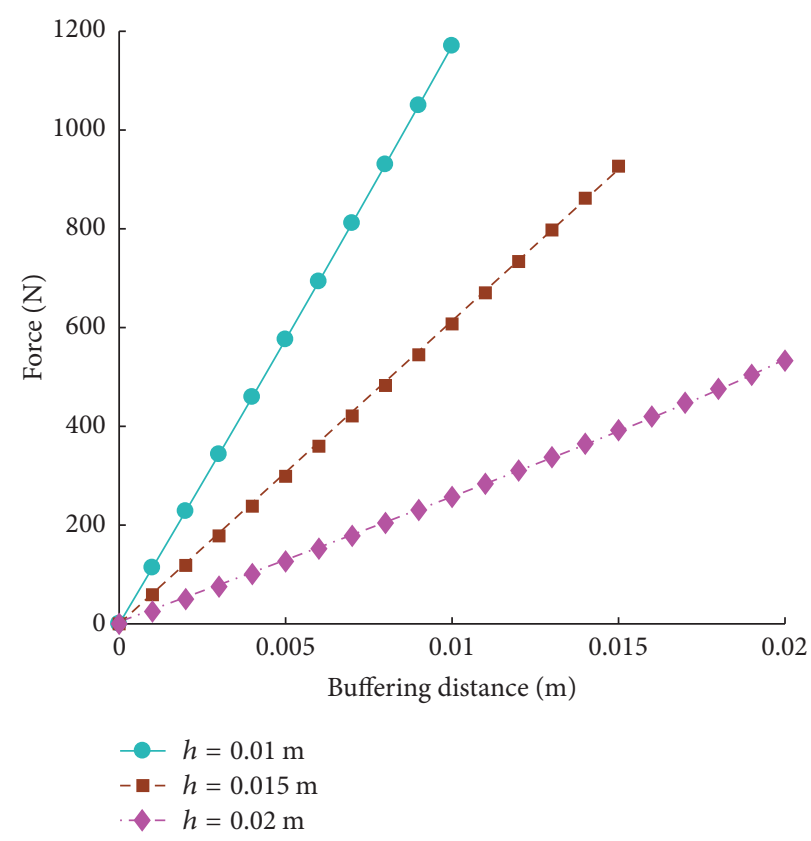

(b)

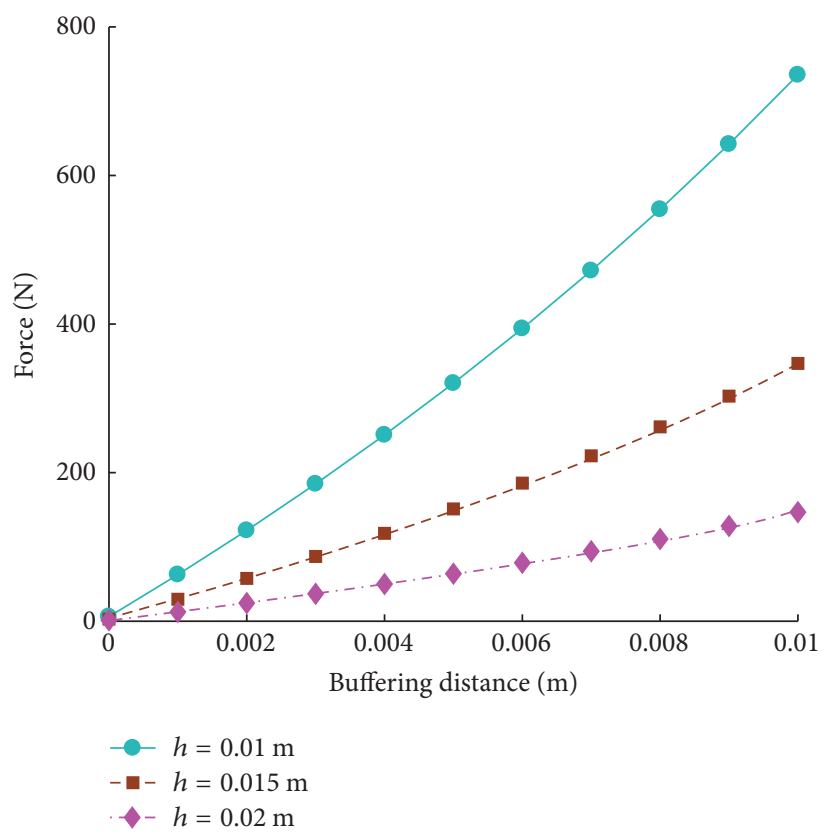

(d)

FIGURE 13: Support force of the ground to the leg. (a) Support force along $x_{0}$-axis with the first walking way. (b) Support force along $y_{0}$-axis with the first walking way. (c) Support force along $x_{0}$-axis with the second walking way. (d) Support force along $y_{0}$-axis with the second walking way.

of the force the trunk suffers more stable in leg state, but it will require the larger torque of the drive motor in the same case because the motor also needs to overcome the deformation. Besides, small stiffness will increase the deformation of the structure. Considering the effect of stiffness on the motion performance when the robot is in wheel state and leg state, the structural stiffness should be considered synthetically.

\section{Experiments}

The control flow chart is seen in Figure 14. When the leg state is needed for the robot, the current state should be determined. If the robot is already in the leg state, the terrain conditions should be determined, and the two different gates can be chosen. On the contrary, if the robot is in the wheel 


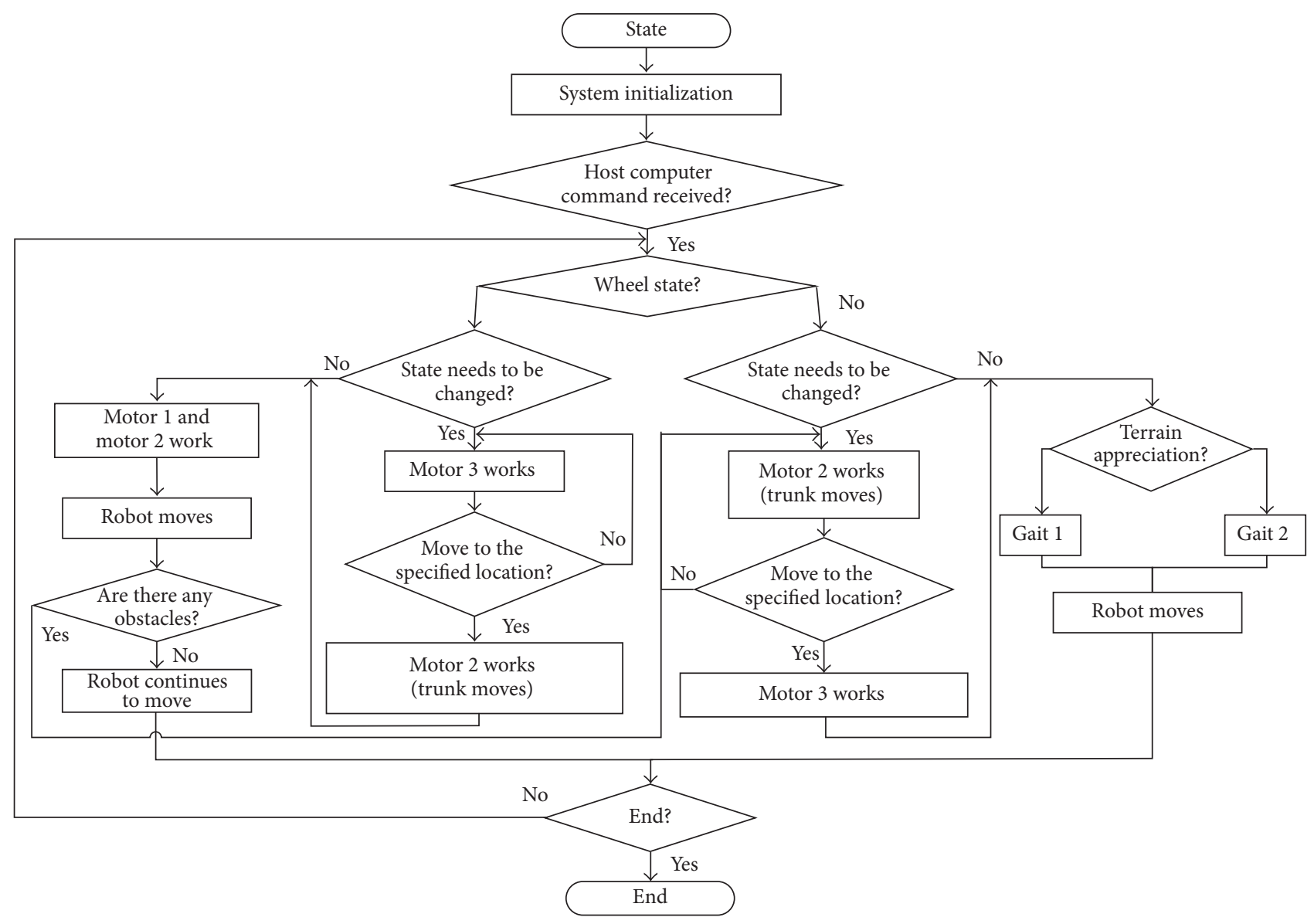

FIGURE 14: Control flow chart.

state, motor 2 works to make the trunk lifted. When the trunk is lifted to the specified position, motor 3 works to make the left half arc structure of the wheel rotate. At this time, the motion state of the robot changes. When the wheel state is needed for the robot and the robot is already in the wheel state, motor 1 and motor 2 work together, and the robot moves forward. When it encounters obstacles, the motion state can be changed from wheel state to leg state. When the wheel state is needed but the robot is in the leg state, the motor works to make the left half arc structure of the wheel rotate, and when the leg structure changes to the wheel structure, motor 2 works to make the center of rotation from the eccentric position to the wheel center. According to the above control method, the robot can realize the conversion of the leg state and wheel state.

The wheel-legged rescue robot is shown in Figure 15. The control hardware is located on the trunk of the robot. Attitude conversion process from wheel state to leg state is shown in Figure 16. First, the trunk of the robot is lifted with the driving of the motor. Then the right half arc structure rotates by the driving of motor 3 until it fits with the left half arc structure. At this time, the rescue robot is in the leg state.

The robot can achieve different leg movement states according to the different terrain environment. When the

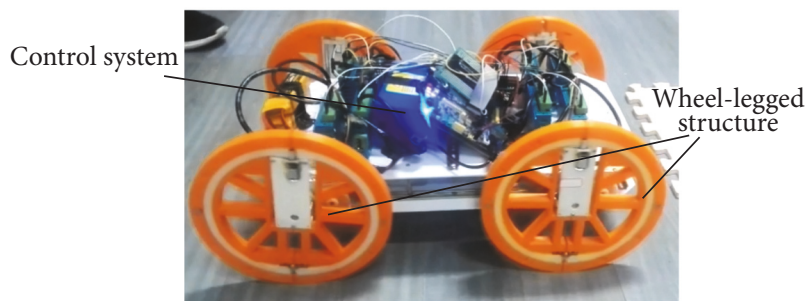

Figure 15: Wheel-legged rescue robot.

robot walks on a rough road, the gait is shown in Figure 17. In the walking process, the two wheel-legged structures which are in a diagonal position move simultaneously. And there are two wheel-legged structures in the support phase, the other two wheel-legged structures in the swing phase. This kind of gait makes the robot move fast, but it cannot get over the high obstacles.

When the robot encounters a high barrier to climb, the gait is shown in Figure 18. The height of the obstacle is $15 \mathrm{~cm}$. First, two wheel-legged structures which are in front of the robot are on the edge of the obstacle in turn. Then the robot climbs the obstacle with the driving of motor 1 and motor 2 . 

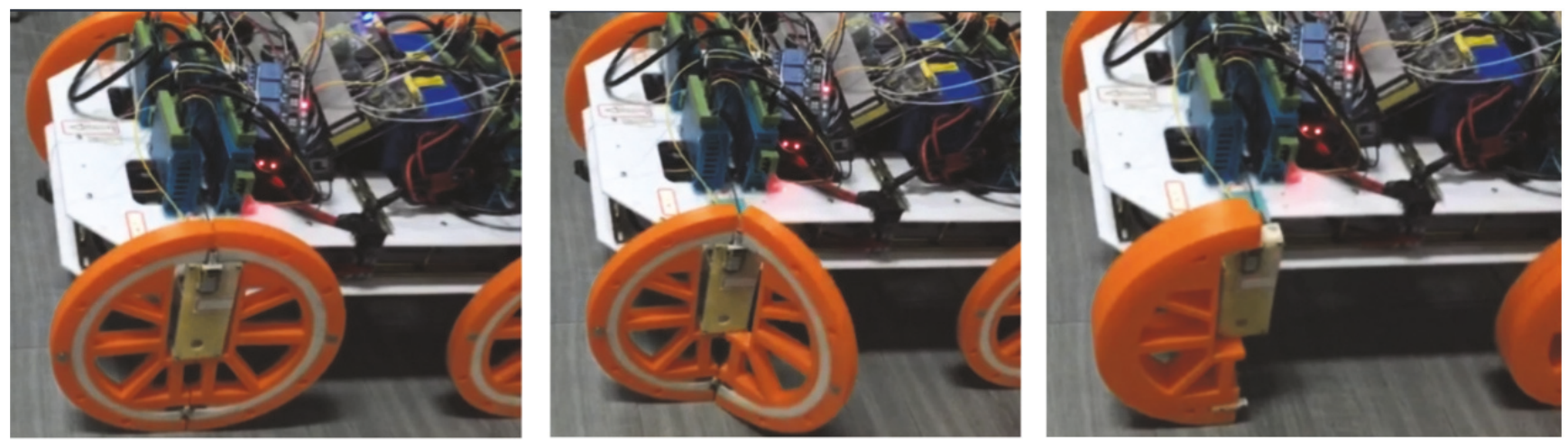

FIGURE 16: Conversion process from wheel state to leg state.
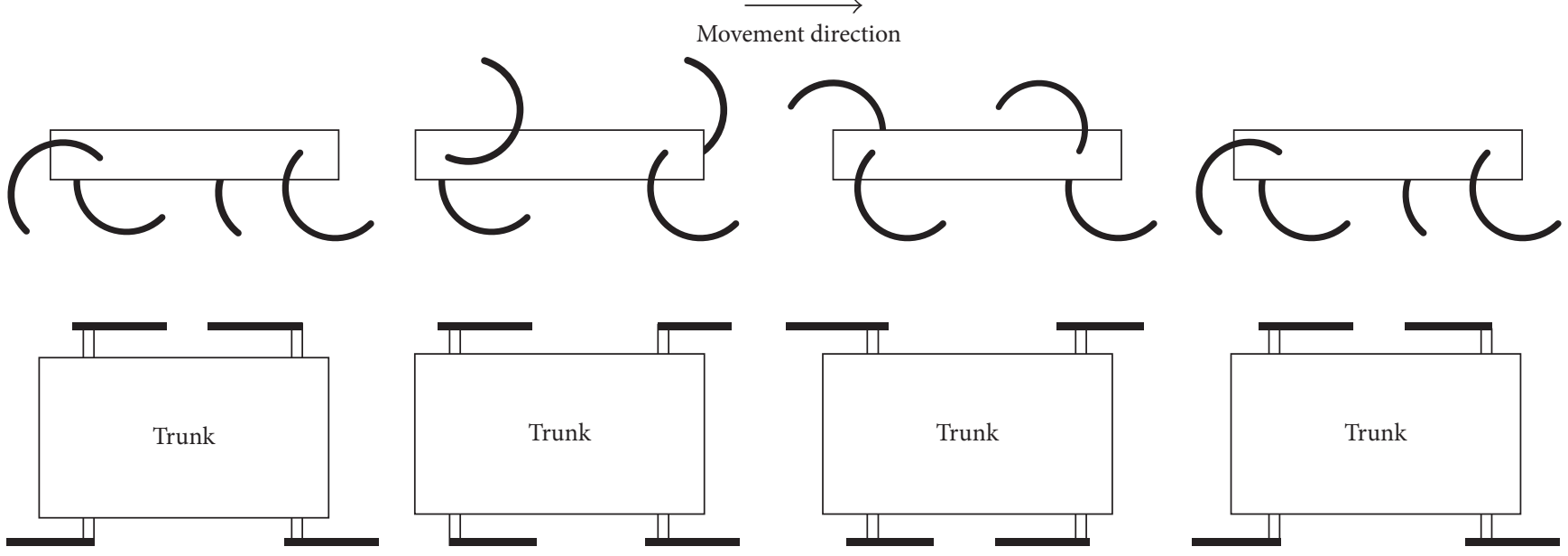

(I)

(II)

(III)

(IV)

(a) Gait sequence diagram

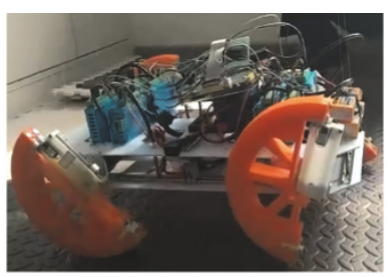

(I)

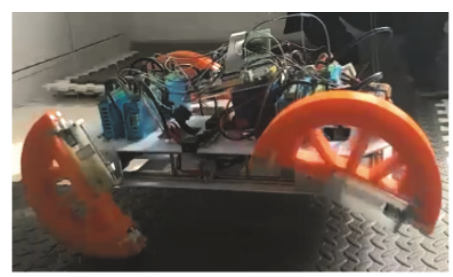

(II)

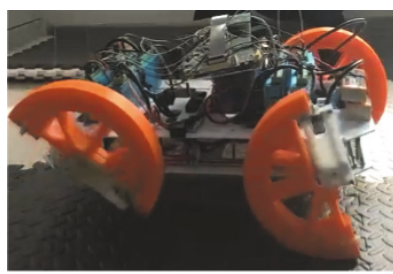

(III)

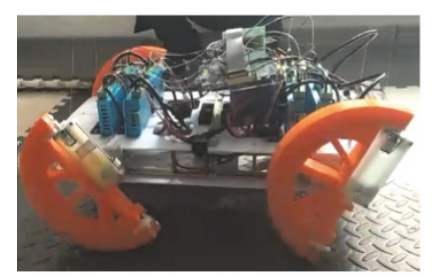

(IV)

(b) Motion diagram of the robot

FIGURE 17: Gait 1 of the robot (walk on rough road).

In particular, the height of the obstruction cannot be higher than the diameter of the wheel. The robot may move slowly with this kind of gait, but the obstacles it can climb can be higher than gait 1 .

In addition, because the wheels are elastic, the robot can reduce the vibration and impact in the rugged environment, and the robot can achieve a smooth movement. Therefore, the robot has good performance and can adapt to the complex and changeable postdisaster environment.

\section{Discussion}

A new wheel-legged rescue robot is designed in this paper. For the mechanical structure, the wheel of the robot can rotate $180^{\circ}$ around the vertical axis and turn into a semicircular structure. Because it does not require any space other than the space occupied by the robot with wheel state during the state change process, it has the advantage of small occupation space. Moreover, compared with the series leg structure and 


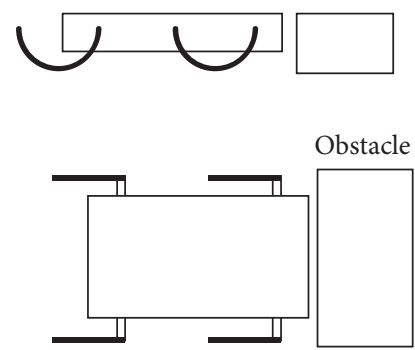

(I)

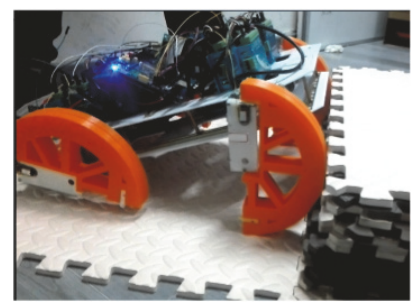

(I)

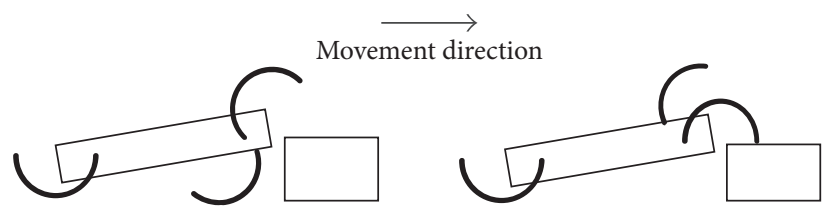

Obstacle

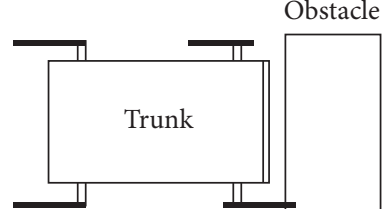

(II)

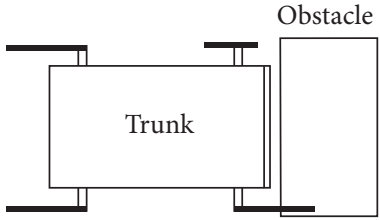

(III)
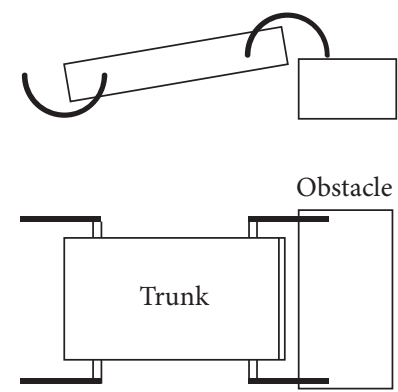

(IV)

(a) Gait sequence diagram

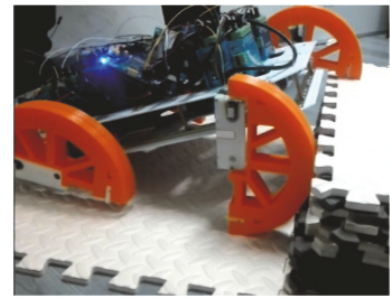

(II)

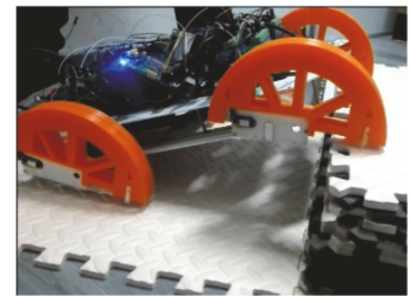

(III)

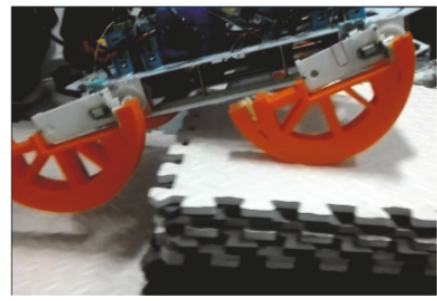

(IV)

(b) Motion diagram of the robot

FIGURE 18: Gait 2 of the robot (overcoming a barrier).

similar parallel leg structure, the structure of semicircle arc leg is simpler and easier to control. This provides a new idea for the design of wheel-legged robot. For the movement state analysis, it provides a reference for motors selection and control. For the structural stiffness analysis, considering the wheel state and leg state share the same structure to contact with the ground and the effects of structural stiffness on the performance of robot with wheel state and leg state are different, the research results can provide a basis for wheel stiffness design. The above research results provide a useful reference for mechanism design, parameter selection, and control strategy.

However, there are still many problems for the wheellegged rescue robot. For example, there is no waterproof structure, intelligent control algorithm is not studied, and the kinematic performance when the robot is in sea wave is not analyzed. All this needs further improvement.

\section{Conclusion}

This paper focuses on the design and analysis for a wheellegged rescue robot. Considering the existing wheel-legged robot has the disadvantages of complex structure, difficult control, large space required for wheel-legged translation, and limited movement environment, a novel wheel-legged rescue robot is designed, and the wheel becomes semicircular leg by the rotation of half wheel along vertical axis. The robot can carry out a variety of motion states and can move on land or in waves. Based on the analysis results of motion states and the effect of structural stiffness of wheel-legged structure on motion performance, the prototype of wheel-legged rescue robot is designed, and the experiments show that the robot has good performance. The present study provided a basis for designing the wheel-legged rescue robot. Further extensions to this work include the design for waterproof structure, research on intelligent control method, and the test in real postdisaster environment.

\section{Conflicts of Interest}

The authors declare that they have no conflicts of interest.

\section{Acknowledgments}

This work was supported by the Natural Science Foundation of Jiangsu Province, China (Grant no. BK20160185), project supported by the National Science Foundation for Young Scientists of China (Grant no. 51705201), the Fundamental Research Funds for the Central Universities (Grant no. JUSRP11718), the Foundation of Jiangsu Key Laboratory of Advanced Food Manufacturing Equipment and Technology (Grant no. FM-2016-08), the Jiangnan University Student's Innovation Training Project, no. 2016343Y, and the National College Student's Innovation Training Program, Project no. 201710295078Y. 


\section{References}

[1] T. Zhang, H. Shang, J. Xu, K. Liu, Y. Zhang, and J. Wang, "Application of robot technology in search and rescue in earthquake ruins," Journal of Natural Disasters, vol. 21, no. 5, pp. 108-112, 2012.

[2] X. P. Dong and X. B. Wang, "Development of rescue robot technology and its application in disaster," Journal of Disaster Prevention \& Mitigation Engineering, vol. 27, no. 1, pp. 112-117, 2007.

[3] H. Lang, M. T. Khan, K.-K. Tan, and C. W. de Silva, "Application of visual servo control in autonomous mobile rescue robots," International Journal of Computers, Communications \& Control, vol. 11, no. 5, pp. 685-696, 2016.

[4] R. R. Murphy, J. Kravitz, S. L. Stover, and R. Shoureshi, "Mobile robots in mine rescue and recovery," IEEE Robotics and Automation Magazine, vol. 16, no. 2, pp. 91-103, 2009.

[5] A. Bodenhamer, B. Pettijohn, J. L. Pezzaniti et al., "3D vision upgrade kit for the TALON robot system," in Proceedings of the Stereoscopic Displays and Applications XXI, vol. 7524, pp. 195211, SPIE - The International Society for Optical Engineering, San Jose, Calif, USA, January 2010.

[6] K. Ito and H. Maruyama, "Semi-autonomous serially connected multi-crawler robot for search and rescue," Advanced Robotics, vol. 30, no. 7, pp. 489-503, 2016.

[7] W. Wang, W. Dong, Y. Su, D. Wu, and Z. Du, "Development of search-and-rescue robots for underground coal mine applications," Journal of Field Robotics, vol. 31, no. 3, pp. 386-407, 2014.

[8] N. Suzuki and Y. Yamazaki, "Basic research on the driving performance of an autonomous rescue robot with obstacles," in Proceedings of the IEEE International Conference on Robotics and Biomimetics, IEEE-ROBIO '15, pp. 982-987, Zhuhai, China, December 2015.

[9] S. Liu and D. Sun, "Minimizing energy consumption of wheeled mobile robots via optimal motion planning," IEEE/ASME Transactions on Mechatronics, vol. 19, no. 2, pp. 401-411, 2014.

[10] Y. Pan, F. Gao, C. Qi, and X. Chai, "Human-tracking strategies for a six-legged rescue robot based on distance and view," Chinese Journal of Mechanical Engineering, vol. 29, no. 2, pp. 219-230, 2016.

[11] P. Wang, J. Li, and Y. Zhang, "The nonfragile controller with covariance constraint for stable motion of quadruped searchrescue robot," Advances in Mechanical Engineering, vol. 2014, Article ID 917381, 10 pages, 2014.

[12] M. Neumann, T. Predki, L. Heckes, and P. Labenda, "Snake-like, tracked, mobile robot with active flippers for urban search-andrescue tasks," Industrial Robot: An International Journal, vol. 40, no. 3, pp. 246-250, 2013.

[13] R. Haraguchi, K. Osuka, S. Makita, S. Tadokoro et al., "The development of the mobile inspection robot for rescue activity, MOIRA2," in Proceedings of the 12th International Conference on Advanced Robotics, ICAR '05, pp. 498-505, Seattle, WA, USA, July 2005.

[14] Z. Zhang, D. Chen, and K. Chen, "Analysis and comparison of three leg models for bionic locust robot based on landing buffering performance," Science China Technological Sciences, vol. 59, no. 9, pp. 1413-1427, 2016.

[15] B. H. Wilcox, "ATHLETE: A cargo and habitat transporter for the moon," in Proceedings of the IEEE Aerospace Conference, pp. 1-7, Montana, Mont, USA, March 2009.

[16] K. Hauser, T. Bretl, J. C. Latombe, and B. Wilcox, "Motion Planning for A Six-Legged Lunar Robot," in Workshop on the
Algorithmic Foundations of Robotics, vol. 47 of Springer Tracts in Advanced Robotics, pp. 301-316, Springer, Berlin, Germany, 2008.

[17] F. B. Amar, V. Budanov, P. Bidaud et al., "A high mobility redundantly actuated mini-rover for self adaptation to terrain characteristics," in Proceedings of the 3rd International Conference on Climbing and Walking Robots, pp. 105-112, Madrid, Spain, 2000.

[18] C. Grand, F. Benamar, and F. Plumet, "Motion kinematics analysis of wheeled-legged rover over 3D surface with posture adaptation," Mechanism and Machine Theory, vol. 45, no. 3, pp. 477-495, 2010.

[19] A. Gronowicz and J. Szrek, "Design of LegVan Wheel-Legged Robots Mechanical and Control System," in SYROM 2009, pp. 145-158, Springer Netherlands, Netherlands, 2010.

[20] A. Suzumura and Y. Fujimoto, "Real-time motion generation and control systems for high wheel-legged robot mobility," IEEE Transactions on Industrial Electronics, vol. 61, no. 7, pp. 36483659, 2014.

[21] X. Ding, K. Li, and K. Xu, "Dynamics and wheel's slip ratio of a wheel-legged robot in wheeled motion considering the change of height," Chinese Journal of Mechanical Engineering, vol. 25, no. 5, pp. 1060-1067, 2012.

[22] X. Wang, X. Wang, H. Yu et al., "Dynamic analysis for the leg mechanism of a wheel-leg hybrid rescue robot," in Proceedings of the 10th UKACC International Conference on Control, CONTROL '14, pp. 504-508, Loughborough, UK, July 2014.

[23] A. Alamdari and V. N. Krovi, "Design of articulated legwheel subsystem by kinetostatic optimization," Mechanism and Machine Theory, vol. 100, pp. 222-234, 2016.

[24] Y. Luo, Q. Li, and Z. Liu, "Design and optimization of wheellegged robot: rolling-wolf," Chinese Journal of Mechanical Engineering, vol. 27, no. 6, pp. 1133-1142, 2014.

[25] R. Siegwart, P. Lamon, T. Estier, M. Lauria, and R. Piguet, "Innovative design for wheeled locomotion in rough terrain," Robotics and Autonomous Systems, vol. 40, no. 2-3, pp. 151-162, 2002.

[26] Y.-S. Kim, G.-P. Jung, H. Kim, K.-J. Cho, and C.-N. Chu, "Wheel transformer: a wheel-leg hybrid robot with passive transformable wheels," IEEE Transactions on Robotics, vol. 30, no. 6, pp. 1487-1498, 2014.

[27] K. Tadakuma, R. Tadakuma, A. Maruyama et al., "Mechanical design of the wheel-leg hybrid mobile robot to realize a large wheel diameter," in Proceedings of the 23rd IEEE/RSJ International Conference on Intelligent Robots and Systems, IROS '10, pp. 3358-3365, Taipei, Taiwan, October 2010.

[28] S.-C. Chen, K.-J. Huang, W.-H. Chen, S.-Y. Shen, C.-H. Li, and P.-C. Lin, "Quattroped: a leg-wheel transformable robot," IEEE/ASME Transactions on Mechatronics, vol. 19, no. 2, pp. 730-742, 2014.

[29] Z. Ma, "Design and simulation of mobile and hopping robot," in Proceedings of the IEEE Workshop on Advanced Robotics and its Social Impacts, IEEE ARSO '16, pp. 273-278, China, July 2016.

[30] Z. Z. Guo, Structure Optimization and Strength Analysis of The Underwater Robot, Northeastern University, Shenyang, China, 2013.

[31] L. S. Pontryagin, Ordinary Differential Equations, AddisonWesley, New York, NY, USA, 1962.

[32] B. W. McCormick, Aerodynamics, Aeronautics and Flight Mechanics, John Wiley and Sons, New York, NY, USA, 2nd edition, 1995. 
[33] G. Y. Pan, Foundation and Application of Automotive Vibration, Peking University Press, Beijing, China, 2013.

[34] L. L. Howell, A. Midha, and T. W. Norton, "Evaluation of equivalent spring stiffness for use in a pseudo-rigid-body model of large-deflection compliant mechanisms," Journal of Mechanical Design, vol. 118, no. 1, pp. 126-131, 1996.

[35] Z. Feng, Y. Yu, and W. Wang, "2R pseudo-rigid-body model of compliant mechanisms with compliant links to simulate tip characteristic," Jixie Gongcheng Xuebao/Journal of Mechanical Engineering, vol. 47, no. 1, pp. 36-42, 2011.

[36] H. J. Su, "A load independent pseudo-rigid-body 3R model for determining large deflection of beams in compliant mechanisms," in Proceedings of the ASME International Design Engineering Technical Conferences Computers and Information in Engineering Conference, pp. 109-121, Brooklyn, NY, USA, 2008. 


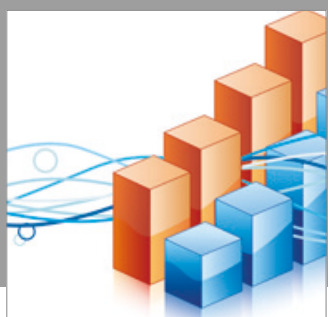

Advances in

Operations Research

vatersals

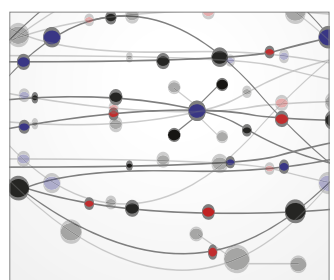

\section{The Scientific} World Journal
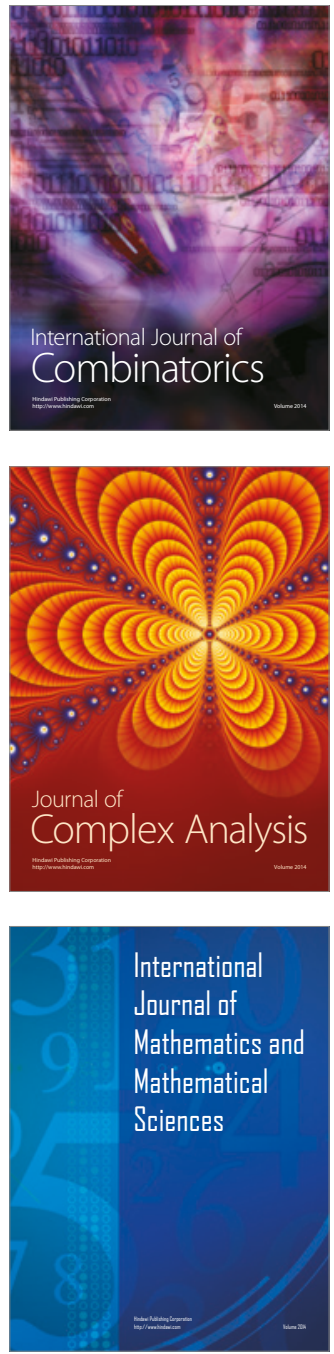
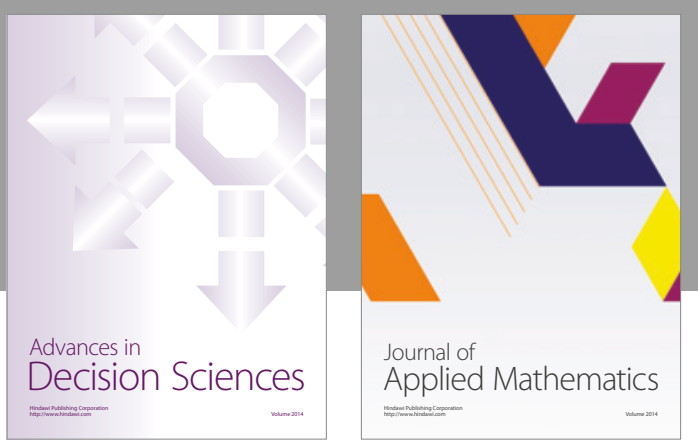

Algebra

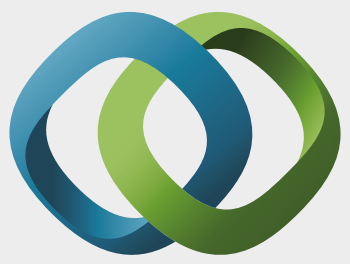

\section{Hindawi}

Submit your manuscripts at

https://www.hindawi.com
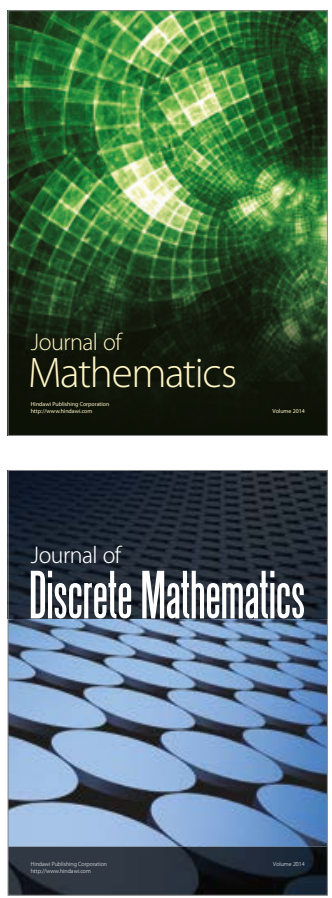

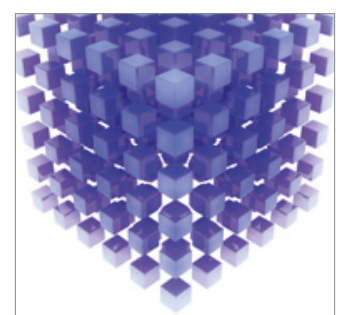

Mathematical Problems in Engineering
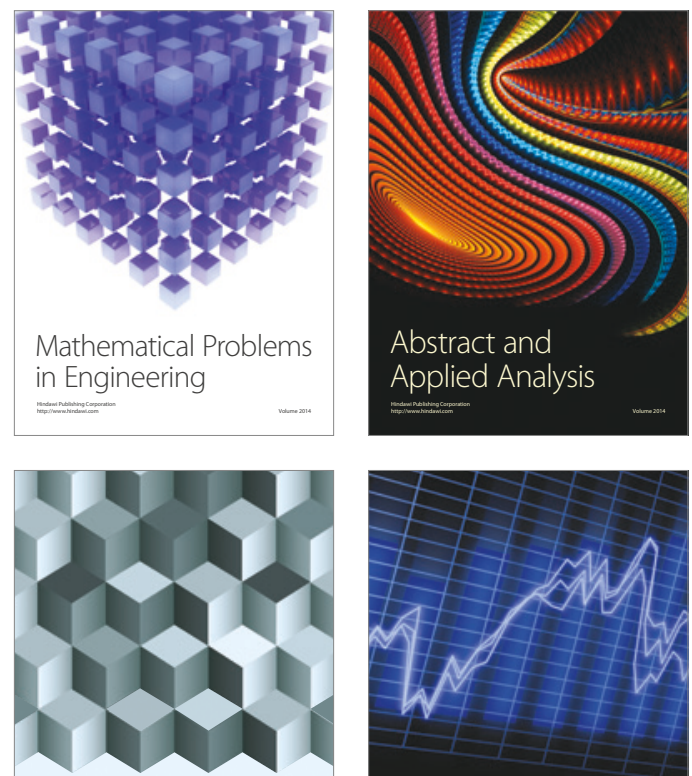

Journal of

Function Spaces

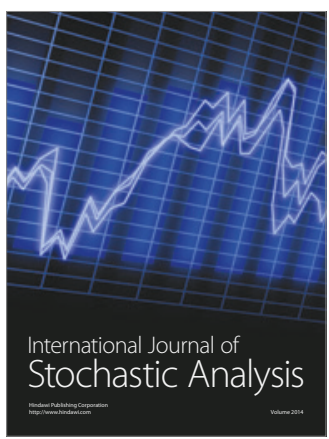

Probability and Statistics
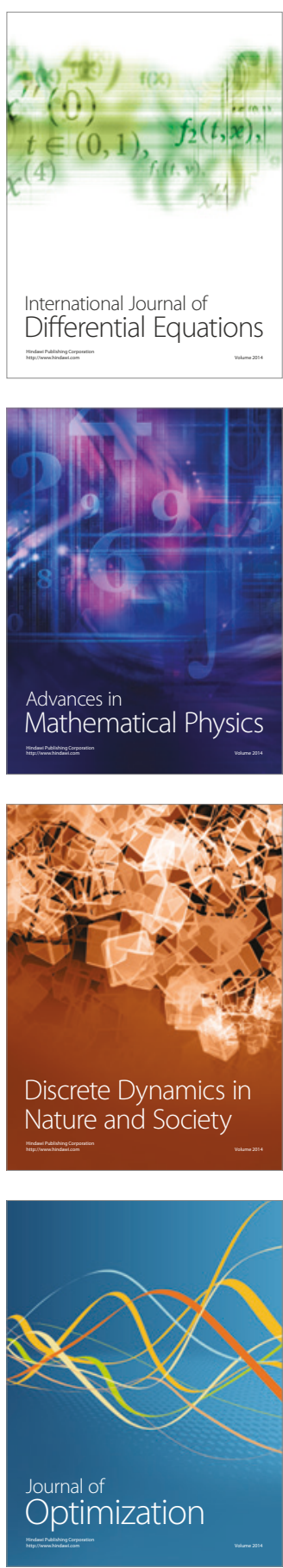